\title{
Five-dimensional vector multiplets in arbitrary signature
}

\section{Gall and T. Mohaupt}

Department of Mathematical Sciences, University of Liverpool, Peach Street, Liverpool L69 7ZL, U.K.

E-mail: Louis.Gall@liverpool.ac.uk, Thomas.Mohaupt@liv.ac.uk

ABSTRACT: We start developing a formalism which allows to construct supersymmetric theories systematically across space-time signatures. Our construction uses a complex form of the supersymmetry algebra, which is obtained by doubling the spinor representation. This allows one to partially disentangle the Lorentz and R-symmetry group and generalizes symplectic Majorana spinors. For the case where the spinor representation is complexirreducible, the R-symmetry only acts on an internal multiplicity space, and we show that the connected groups which occur are $\mathrm{SO}(2), \mathrm{SO}_{0}(1,1), \mathrm{SU}(2)$ and $\mathrm{SU}(1,1)$.

As an application we construct the off-shell supersymmetry transformations and supersymmetric Lagrangians for five-dimensional vector multiplets in arbitrary signature $(t, s)$. In this case the R-symmetry groups are $\mathrm{SU}(2)$ or $\mathrm{SU}(1,1)$, depending on whether the spinor representation carries a quaternionic or para-quaternionic structure. In Euclidean signature the scalar and vector kinetic terms differ by a relative sign, which is consistent with previous results in the literature and shows that this sign flip is an inevitable consequence of the Euclidean supersymmetry algebra.

KeYwords: Extended Supersymmetry, Space-Time Symmetries, String Duality

ARXIV EPRINT: 1805.06312 


\section{Contents}

1 Introduction and summary of results 1

2 Supersymmetry and bilinear forms 3

2.1 Clifford algebras and spinors 4

2.2 Bilinear forms, supersymmetry, and R-symmetry groups 6

$\begin{array}{lll}2.2 .1 & \text { Supersymmetry algebras and bilinear forms } & 6\end{array}$

2.2.2 Standard bilinear forms 8

2.2.3 R-symmetry groups $\quad 8$

2.3 Doubled spinors $\quad 9$

2.4 Reality conditions $\quad 10$

$\begin{array}{lll}2.4 .1 & \text { Symplectic Majorana spinors } & 10\end{array}$

2.4.2 Real doubled spinors defined by a Majorana condition 11

$\begin{array}{ll}2.5 & \text { Determination of R-symmetry groups } \\ \end{array}$

3 Minimal supersymmetry in five dimensions $\quad \mathbf{1 6}$

3.1 Real doubled spinors in $(0,5),(1,4),(4,1)$ and $(5,0)$ - using the symplectic $\begin{array}{ll}\text { Majorana condition } & 18\end{array}$

3.2 Real doubled spinors in $(2,3)$ and $(3,2)$ - using the Majorana condition $\quad 19$

3.3 Reality properties of spinor bilinears and R-group tensors 20

3.4 No $\mathcal{N}=1$ superymmetry algebras in signatures $(2,3)$ and $(3,2) \quad 22$

4 Five-dimensional vector multiplets $\quad 22$

$\begin{array}{lll}4.1 & \text { Supersymmetry variations } & 23\end{array}$

4.2 Supersymmetric Lagrangians 24

4.3 Overview and discussion of supersymmetry variations and Lagrangians by $\begin{array}{ll}\text { signature } & 27\end{array}$

$\begin{array}{lll}\text { 4.3.1 Signature }(1,4) & 27\end{array}$

$\begin{array}{ll}4.3 .2 & \text { Signature }(4,1) \\ 4.3 .3 & \text { Signature }(0,5)\end{array}$

$\begin{array}{lll}4.3 .3 & \text { Signature }(0,5) & 28\end{array}$

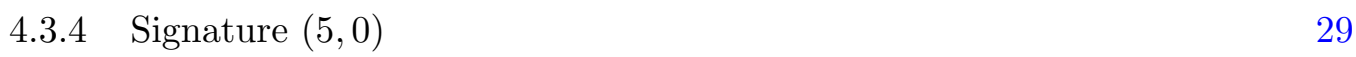

$\begin{array}{lll}4.3 .5 & \text { Signature }(2,3) & 29\end{array}$

$\begin{array}{ll}4.3 .6 & \text { Signature }(3,2) \\ \end{array}$

5 Open problems and outlook 30

$\begin{array}{ll}\text { A Spinor conventions } & 31\end{array}$

B Para-quaternions $\quad 32$

C Quaternions $\quad 34$ 


\section{Introduction and summary of results}

Non-Lorentzian space-time signatures are relevant for a variety of reasons. For Euclidean signature this is obvious, since the study of non-perturbative effects, such as instantons, makes use of the Euclidean functional integral formalism. Moreover, the stationary sectors of Lorentzian theories can be studied through effective Euclidean theories obtained by dimensional reduction over time. In quantum gravity one might also ask whether the Lorentzian signature of space-time is to be accepted as 'given,' or whether it needs to be explained, for example within a framework where signature change occurs dynamically. In string theory the study of the web of dualities connecting different types of string theories naturally leads to the inclusion of exotic, multi-time signatures once T-duality transformations with respect to time are considered [1-3]. To facilitate such studies, a systematic way to construct and relate theories, in particular supersymmetric theories, across space-time signatures is needed. One common approach to changing space-time signature, is the ad hoc flipping of signs and insertion of factors of $i$. A more systematic and well developed way to obtain Euclidean theories is to start with a supersymmetric theory in Lorentzian signature and then to dimensionally reduce over time [4-14]. While this guarantees one finds a theory invariant under Euclidean supersymmetry, it excludes theories that cannot be obtained by dimensional reduction. Moreover, only in a few cases $[6,14]$ have these reductions been carried out in an off-shell formulation and have fully included the fermions. In most cases attention has been restricted to on-shell formulations and to bosonic terms, while the fermions have been neglected or only been considered through the Killing spinor equations evaluated on bosonic backgrounds. In [13] analytic continuation of the Killing spinor equations were used to obtain the supersymmetry variations of the fermions, and, by imposing closure of the algebra, the bosonic terms of the on-shell Lagrangian for five-dimensional vector multiplets coupled to supergravity for all signatures.

A systematic construction should start with the supersymmetry algebra, then where possible construct an off-shell representation of the algebra on fields, and only then proceed to invariant Lagrangians. The natural way to obtain a universal construction that allows to treat all signatures in a given dimension simultaneously is to work with the complex form of the supersymmetry algebra, to construct its representations on complexified fields, and finally a 'holomorpic master Lagrangian.' To specialize to particular signatures one imposes suitable reality conditions and obtains the corresponding field representations and Lagrangians. In this paper we start developing such a formalism and demonstrate its viability by applying it to the case of rigid five-dimensional off-shell vector multiplets. Our approach makes use of the relation between Poincaré Lie superalgebras and real, symmetric, $\mathrm{Spin}_{0}$-equivariant vector-valued bilinear forms. This allows us to use results from the mathematical literature, in particular the classification Poincaré Lie superalgebras in arbitrary dimension and signature [15]. While in spirit our approach is similar to the treatment of maximal supergravity theories in dimensions 10 and 11 in $[16,17]$, one difference is that we do not need to assume that the supersymmetry algebra arises as a contraction of an ortho-symplectic Lie superalgebra. This is important since it is not clear a priori whether all Poincaré Lie superalgebras (in all signatures) can be obtained as contractions. BPS- 
charges, also called polyvector charges, or, by abuse of terminology, central charges, which in the ortho-symplectic framework arise through different contractions, can be added to our formalism using the work of [18], though we will leave this aspect to future work.

The core piece of formalism that we develop in the first part of this paper is what we call the doubled spinor module. This accounts for the doubling, or complexification, of the supercharges and fermionic fields that characterizes the complex form of the theory. As we will explain in more detail in the paper, the essential part of defining a supersymmetry algebra is to choose a so-called admissible bilinear form on the spinor module $\mathbf{S}$ of the Poincaré Lie superalgebra $\mathfrak{s p}(V)=V+\mathfrak{s} \mathfrak{o}(V)+\mathbf{S}$, where $V=\mathbb{R}^{t, s}$ is a space-time with signature $(t, s)$. In short, the admissible bilinear form determines the superbracket between the supercharges of the theory. The complex form of the theory is defined using the doubled spinor space $\mathbf{S} \oplus \mathbf{S}$, which is equipped with a complex, symmetric vector-valued bilinear form, which is equivariant with respect to the complex Spin group, and thus gives rise to a superbracket for the complex form of the supersymmetry algebra. Real forms are obtained by picking a $\operatorname{Spin}(V)$-invariant real structure on $\mathbf{S} \oplus \mathbf{S}$ and restricting to the real points $(\mathbf{S} \oplus \mathbf{S})^{\rho} \simeq \mathbf{S}$. The restriction of the complex bilinear form gives rise to the real bilinear form associated to the real superbracket. A well known example of reformulating spinors by first doubling and then imposing a reality condition are symplectic Majorana spinors, which are naturally included in our formalism. One advantage of the doubling is that part of the R-symmetry group becomes manifest when writing the doubled spinor module as a complex tensor product, $\mathbf{S} \oplus \mathbf{S} \simeq \mathbf{S} \otimes \mathbb{C}^{2}$. In this product form, the Lorentz Lie algebra only acts on the first factor, whereas the R-symmetry acts in general on both $\mathbf{S}$ and on the multiplicity space $\mathbb{C}^{2}$. In the simplest case, where $\mathbf{S}$ is complex irreducible, that is for Dirac spinors in odd dimensions, Schur's lemma implies that the R-symmetry group can be taken to only act on $\mathbb{C}^{2}$. In this case the R-symmetry group of the complex theory is either $O(2, \mathbb{C})$ or $\operatorname{Sp}(2, \mathbb{C})$, depending on whether the complex bilinear form is symmetric or antisymmetric when restricted to $\mathbb{C}^{2}$. Since the reality condition selects a real form of the complex R-symmetry group, it follows that the connected part of the real Rsymmetry group must be $\mathrm{SO}(2), \mathrm{SO}_{0}(1,1), \mathrm{USp}(2) \simeq \mathrm{SU}(2)$ or $\mathrm{Sp}(2, \mathbb{R}) \simeq \mathrm{SU}(1,1)$. In the general case, where $\mathbf{S}$ is complex reducible, these groups are part of a larger R-symmetry group. In this paper we will work out the R-symmetry groups for supersymmetry algebras based on complex irreducible spinor modules, while the general case will be presented in a separate paper [19].

Having developed the general formalism to this point, we then specialize to five dimensions and to vector multiplets. In five dimensions the complex spinor module $\mathbb{S}$, that is the representation by Dirac spinors, is both complex and real irreducible in Lorentzian signature and defines the unique minimal supersymmetry algebra. Counting supercharges in multiples of the minimal four-dimensional Lorentzian signature supersymmetry algebra, we refer to this algebra as the five-dimensional $\mathcal{N}=2$ supersymmetry algebra. While for signatures $(2,3)$ and $(3,2)$ Dirac spinors are real reducible, that is, one can define Majorana spinors, we show that there is no corresponding $\mathcal{N}=1$ supersymmetry algebra with four real supercharges, since the superbracket is trivial. Thus there is a unique minimal supersymmetry algebra with eight real supercharges for all five-dimensional signatures. We 
then carry out the programme outlined above and obtain the off-shell field representations and invariant Lagrangians of vector multiplets for all signatures $(5,0), \ldots,(0,5)$. From our results it is manifest that theories which are space-time mirrors, that is related by $t \leftrightarrow s$, are physically equivalent in the sense that all sign flips and factors $i$ are determined by going between a mostly plus and a mostly minus convention for the space-time metric, plus taking into account the signature dependence of the reality properties of spinor bilinears. Throughout the paper we adopt the convention of referring to the smaller of the numbers $t, s$ as time, so that there are at most two time-like dimensions. In the Euclidean signatures $(0,5)$ and $(5,0)$ we find that the scalar and vector terms always come with a relative sign. This confirms the indirect arguments given in [12] and shows that this relative sign follows inevitably from the Euclidean supersymmetry algebra. Note that this is not clear a priori. In particular, it has been argued that a similar sign flip for four-dimensional vector multiplets can be removed by a field redefinition $[11,12]$. We also compare our results to those of [13], where the bosonic on-shell Lagrangians for five-dimensional vector multiplets coupled to supergravity have been obtained for all signature by analytic continuation of the Killing spinor equations of the Lorentzian theory. We find that all signature dependent relative signs agree.

The outline of this paper is as follows. In section 2 we first present the background on Clifford algebras and spinors needed to make this paper self-contained. Then we review the relation between supersymmetry algebras and vector-valued bilinear forms. We introduce the doubled spinor construction and analyse which R-symmetry groups can occur for supersymmetry algebras based on complex irreducible spinors. In section 3 we turn to minimal supersymmetry in five dimensions, where the relevant spinor representation $\mathbf{S}$ is the one by Dirac spinors denoted $\mathbb{S}$. We show explicitly how the superbrackets formulated on the complex spinor module $\mathbb{S}$ and on the doubled spinor module $\mathbb{S} \otimes \mathbb{C}^{2}$ are related, and show that while Majorana spinors can be defined for some signatures, the corresponding $\mathcal{N}=1$ supersymmetry algebras are trivial. In section 4 we construct an off-shell representation of the complex supersymmetry algebra by vector multiplets, and a corresponding invariant off-shell Lagrangian. By imposing reality conditions, we obtain the transformations and Lagrangians for all signatures from $(0,5)$ to $(5,0)$. In section 5 we give a brief outlook onto open problems and future directions. Some material has been relegated to appendices in order not to avoid breaking the flow of the presentation. While we suppress spinor indices most of the time, we need to give the details which allow us to translate between vector-valued bilinear forms and supercharge anticommutators. This is done in appendix A. Appendices B and C contain the relevant background on para-quaternions and quaternions.

\section{Supersymmetry and bilinear forms}

In this section we work in arbitrary dimension and signature. After providing the background on Clifford algebras, supersymmetry algebras, and admissible bilinear forms needed to make this article self-contained, we introduce the formalism based of doubled spinors, which allows to define a complex form of the supersymmetry algebra and then to specialize 
to any signature by imposing reality conditions. We review the useful concepts of the Schur algebra and Schur group, and investigate how the choice of a reality condition determines the R-symmetry group of the real superalgebra.

\subsection{Clifford algebras and spinors}

We work on a 'space-time' $V=\mathbb{R}^{t, s}$ of signature $(t, s)$ with metric

$$
\eta=\operatorname{diag}(\underbrace{-1,-1 \ldots,}_{t} \underbrace{+1,+1, \ldots}_{s})
$$

and isometry Lie algebra $\mathfrak{p}(V)=V+\mathfrak{s o}(V)$, where $V=\mathbb{R}^{t, s}$ are the translations ${ }^{1}$ and $\mathfrak{s o}(V)=\mathfrak{s o}(t, s)$ the linear transformations preserving $\eta$. Space-time indices are denoted $\mu, \nu=1, \ldots, t+s$. The real Clifford algebra $C l(V)=C l_{t, s}$ has generators $\gamma_{\mu}$, with defining relations

$$
\left\{\gamma_{\mu}, \gamma_{\nu}\right\}=2 \eta_{\mu \nu}
$$

Lorentz indices on Clifford generators are raised and lowered using $\eta$, that is $\gamma^{\mu}=\eta^{\mu \nu} \gamma_{\nu}$. Our conventions are the same as in [6]. Note that they differ from the conventions in [20] by a relative sign in the definining relation and another sign in the definition of $\eta$. The combined effect of both relative signs is that $C l_{t, s}$ refers to the same associative real algebra. While our notation is adapted to the 'mostly plus' convention for Minkowski space-time, we will interpret $\min \{t, s\}$ as the number of time-like directions, because we expect that theories related by an overall sign change, $t \leftrightarrow s$ of the metric are physically equivalent. Thus, for example, in four and five dimensions there are at most two time-like directions.

We use a representation of the Clifford generators in terms of complex square matrices of size $2^{[(t+s) / 2]}$, with the following Hermiticity properties:

$$
\left(\gamma^{\mu}\right)^{\dagger}= \begin{cases}-\gamma^{\mu}, & \text { for } \mu=1, \ldots, t \\ \gamma^{\mu}, & \text { for } \mu=t+1, \ldots, t+s .\end{cases}
$$

This fixes the $\gamma$-matrices up to unitary equivalence. The $\gamma$-matrices provide an irreducible representation of the complex Clifford algebra $\mathbb{C l}_{t+s}=C l_{t, s} \otimes \mathbb{C}$. By restriction to a representation of the real group $\operatorname{Spin}(t, s) \subset C l_{t, s} \subset \mathbb{C} l_{t+s}$ one obtains a complex spinor representation of the Lorentz group, the representation by Dirac spinors. The corresponding representation space will be referred to as the complex spinor module $\mathbb{S}$. Dirac spinors provide an irreducible complex $\operatorname{Spin}(t, s)$ - representation for odd $t+s$, while for even $t+s$ Dirac spinors decompose into Weyl spinors, $\mathbb{S}=\mathbb{S}_{+} \oplus \mathbb{S}_{-}$.

The $\gamma$-matrices can be related to the Hermitian conjugate, complex conjugate and transposed matrices by relations of the form $[6]^{2}$

$$
\begin{aligned}
\left(\gamma^{\mu}\right)^{\dagger} & =(-1)^{t} A \gamma^{\mu} A^{-1}, \\
\left(\gamma^{\mu}\right)^{*} & =(-1)^{t} \tau B \gamma^{\mu} B^{-1}, \\
\left(\gamma^{\mu}\right)^{T} & =\tau C \gamma^{\mu} C^{-1},
\end{aligned}
$$

\footnotetext{
${ }^{1}$ We do not distinguish by notation between the vector space with inner product $V=\mathbb{R}^{t, s}=\left(\mathbb{R}^{t+s}, \eta\right)$, the associated affine space, and the translation group $\left(\mathbb{R}^{t+s},+\right)$ acting on the affine space.

${ }^{2}$ See also [21] for a comprehensive review. Note that the parameters $\epsilon$ and $\eta$ used there are related to our parameters by $\sigma=-\epsilon$ and $\tau=-\eta$.
} 
where $\tau= \pm 1$. For $A$ we make the explicit choice

$$
A=\gamma_{1} \cdots \gamma_{t},
$$

which implies

$$
A^{\dagger}=(-1)^{\frac{t(t+1)}{2}} A, \quad A^{-1}=(-1)^{t} \gamma_{t} \cdots \gamma_{1} .
$$

The matrix $A$ generalizes the ' $\Gamma_{0}$-matrix' familar from Lorentz signature and enters into the definition of Dirac spinor bilinears, and of the Dirac conjugate of a spinor, $\bar{\psi}^{(D)}=\psi^{\dagger} A$. The matrix $C$ is the charge conjugation matrix, which relates particle and anti-particle states, and defines the Majorana conjugate $\bar{\psi}=\psi^{T} C$ of spinors. It can be chosen to satisfy (see for example [21])

$$
C^{-1}=C=C^{\dagger}
$$

$C$ is either symmetric or antisymmetric,

$$
C^{T}=\sigma C,
$$

where $\sigma= \pm 1$. Which values of $\sigma$ and $\tau$ can occur depends on the dimension. For each value of $\tau$ there is always precisely one corresponding value of $\sigma$. In even dimensions, $t+s=2 n$, both $\tau=1$ and $\tau=-1$ are possible, and there are two inequivalent choices for $C$, denoted $C_{-\tau}$. In odd dimensions, $t+s=2 n+1$, the matrix $C$ is unique up to equivalence, and $\tau=(-1)^{(t+s-1) / 2)}$.

Given $A$ and $C$, the matrix $B$ can be chosen as

$$
B=\left(C A^{-1}\right)^{T} .
$$

Then $B$ is unitary, $B^{\dagger} B=11$, and

$$
B B^{*}= \pm 11
$$

where the sign depends on the signature, and, in even dimensions, on the choice of the charge conjugation matrix $C=C_{ \pm}$. In signatures where it is possible to choose $B B^{*}=+1$, the matrix $B$ can be used to impose a reality condition and to define Majorana spinors. Mathematically, $B$ defines a family of real structures on the complex spinor module $\mathbb{S}$,

$$
\rho_{\mathbb{S}}^{(\alpha)}(\lambda)=\alpha^{*} B^{*} \lambda^{*}, \quad\left(\rho_{\mathbb{S}}^{(\alpha)}\right)^{2}=11,
$$

where $\alpha \in \mathbb{C}$ is a phase, $|\alpha|=1$. The presence of this phase reflects that multiplying $B$ by a phase does not change the relations $B B^{\dagger}=11$ and $B B^{*}= \pm 1$. We have made an explicit choice for $B$ in terms of $A$ and $C$, but will use the freedom represented by $\alpha$ later to choose our reality conditions such that the resulting formulae are uniform for all signatures. The real structure defined by $B$ is Spin $_{0}$-invariant: ${ }^{3}$

$$
\rho_{\mathbb{S}}^{(\alpha)}\left(\gamma^{\mu} \lambda\right)=\alpha^{*} B^{*}\left(\gamma^{\mu}\right)^{*} \lambda^{*}=(-1)^{t} \tau \gamma^{\mu} B^{*} \lambda^{*},
$$

\footnotetext{
${ }^{3}$ That is, it is invariant under the Lie algebra $\mathfrak{s o}(V)$, and hence under the connected component of the Spin group.
} 
and therefore

$$
\rho_{\mathbb{S}}^{(\alpha)}\left(\gamma^{\mu \nu} \lambda\right)=\alpha^{*} B^{*}\left(\gamma^{\mu \nu}\right)^{*} \lambda^{*}=(-1)^{2 t} \tau^{2} \gamma^{\mu \nu} \alpha^{*} B^{*} \lambda^{*}=\gamma^{\mu \nu} \rho_{\mathbb{S}}^{(\alpha)}(\lambda) .
$$

Majorana spinors $\lambda \in \mathbb{S}_{M} \subset \mathbb{S}$ are by definition the spinors which are invariant under the real structure,

$$
\rho_{\mathbb{S}}^{(\alpha)}(\lambda)=\lambda \Leftrightarrow \lambda^{*}=\alpha B \lambda
$$

In signatures where $B B^{*}=-1$, the matrix $B$ defines a family of Spino-invariant quaternionic structures,

$$
j_{\mathbb{S}}^{(\alpha)}(\lambda)=\alpha^{*} B^{*} \lambda^{*}, \quad\left(j_{\mathbb{S}}^{(\alpha)}\right)^{2}=-11 .
$$

The Spin $_{0}$-invariance holds for the same reason as for the real structure: we have $j_{\mathbb{S}}^{(\alpha)}\left(\gamma^{\mu} \lambda\right)= \pm \gamma^{\mu} j_{\mathbb{S}}^{(\alpha)}(\lambda)$, and therefore $j_{\mathbb{S}}^{(\alpha)}\left(\gamma^{\mu \nu} \lambda\right)=\gamma^{\mu \nu} j_{\mathbb{S}}^{(\alpha)}(\lambda)$. While a quaternionic structure cannot be used to define a reality condition on $\mathbb{S}$ itself, it can be used to impose a reality condition on complex spaces of the form $\mathbb{S} \otimes V_{\mathbb{C}}$, where $V_{\mathbb{C}}$ is a complex vector space equipped with a quaternionic structure. Symplectic Majorana spinors are one example of such a construction and will be discussed later.

We remark that the complex spinor module $\mathbb{S} \simeq \mathbb{C}^{2 n}$ can always either be viewed as a vector space $\mathbb{S} \simeq \mathbb{H}^{n}$ over the skew field $\mathbb{H}$ of quaternions, or as a module $\mathbb{H}^{\prime n}$ over the ring of para-quaternions $\mathbb{H}^{\prime}{ }^{4}$ The reason is that the $\operatorname{Spin}_{0}$-invariant endomorphism defined by

$$
J=\left\{\begin{array}{l}
j_{\mathbb{S}}^{(\alpha)}, \text { if } \mathbb{S} \text { has an invariant quaterionic structure } \\
\rho_{\mathbb{S}}^{(\alpha)}, \text { if } \mathbb{S} \text { has an invariant real structure }
\end{array}\right.
$$

anti-commutes with the natural complex structure $I$, which acts through multiplication by the complex unit, $I: \lambda \mapsto i \lambda$. Therefore $K=I J=-J I$ is another $\operatorname{Spin}_{0}$-invariant endomorphism which anti-commutes with $I$ and $J$. If $B B^{*}=\varepsilon 11, \varepsilon= \pm 1$ then the three endomorphisms $I, J, K$ satisfy

$$
I^{2}=\varepsilon J^{2}=\varepsilon K^{2}=-11, \quad I, J, K \text { pairwise anticommuting, }
$$

which is the quaternionic algebra for $\varepsilon=1$ and the para-quaternionic algebra for $\varepsilon=-1$.

\subsection{Bilinear forms, supersymmetry, and R-symmetry groups}

\subsubsection{Supersymmetry algebras and bilinear forms}

Supersymmetry extends the Poincaré Lie algebra $\mathfrak{p}(V)=V+\mathfrak{s o}(V)$ to a Poincaré Lie superalgebra $\mathfrak{s p}(V)=\mathfrak{g}_{0}+\mathfrak{g}_{1}$, where the even part $\mathfrak{g}_{0}$ is the Poincaré Lie algebra, and the odd part $\mathfrak{g}_{1}$ is a spinor module $\mathbf{S}$ of $\mathfrak{s o}(V)$, which may be reducible or irreducible. The Poincaré Lie superalgebra is completely determined once we specify the supersymmetry algebra (supertranslation algebra)

$$
\left\{Q_{\alpha}, Q_{\beta}\right\}=K_{\alpha \beta}^{\mu} P_{\mu},
$$

\footnotetext{
${ }^{4}$ We refer to the appendices for brief reviews of the relevant facts about quaternions and para-quaternions.
} 
where the translation generators $P_{\mu}$ span $V$, while the supercharges $Q_{\alpha}$ span $\mathbf{S}$. Since the bracket $\{\cdot, \cdot\}$ is symmetric, the structure constants $K_{\alpha \beta}^{\mu}$ can be interpreted as representing a real $\mathbf{S p i n}_{0}$-equivariant symmetric vector-valued bilinear form on the spinor module $\mathbf{S}$ :

$$
K: \operatorname{Sym}(\mathbf{S} \otimes \mathbf{S}) \rightarrow \mathbb{R}^{t, s}: \quad(\lambda, \chi) \mapsto K^{\mu}(\lambda, \chi)=K^{\mu}(\chi, \lambda) .
$$

Conversely, any real Spin 0 -equivariant, symmetric vector-valued bilinear form defines a superbracket. The anti-commutation relations (2.5) are obtained by expanding spinors in the basis provided by the supercharges $Q_{\alpha}$ :

$$
\left\{\lambda^{\alpha} Q_{\alpha}, \chi^{\beta} Q_{\beta}\right\}=K^{\mu}(\lambda, \chi) P_{\mu}=\lambda^{\alpha} \chi^{\beta} K_{\alpha \beta}^{\mu} P_{\mu} .
$$

Note that in this approach there is no need to explicitly check the super Jacobi identity, because it holds automatically. It was shown in [15] how all supersymmetry algebras in all dimensions and signatures can be constructed from vector-valued bilinear forms. In the following we draw on results of this work to explain how such vector-valued bilinear forms can be constructed using so-called admissible bilinear forms. We will not consider central charges and other BPS charges, which correspond to polyvector extensions of Poincaré Lie superalgebras, but remark that these extensions have likewise been constructed and classified using the language of admissible bilinear forms [18].

Starting from a bilinear form

$$
\beta: \mathbf{S} \otimes \mathbf{S} \rightarrow \mathbb{R}
$$

on a spinor module $\mathbf{S}$, one can define a vector-valued bilinear form by inserting a Clifford generator,

$$
K^{\mu}(\lambda, \chi):=\beta\left(\gamma^{\mu} \lambda, \chi\right)
$$

One defines the symmetry $\sigma(\beta)= \pm 1$ and type $\tau(\beta)= \pm 1$ of a bilinear form as follows:

$$
\begin{aligned}
& \sigma(\beta): \beta(\lambda, \chi)=\sigma(\beta) \beta(\chi, \lambda), \\
& \tau(\beta): \beta\left(\gamma^{\mu} \lambda, \chi\right)=\tau(\beta) \beta\left(\lambda, \gamma^{\mu} \chi\right) .
\end{aligned}
$$

Note that the quantities $\sigma, \tau$ introduced earlier are the symmetry $\sigma(C)$ and type $\tau(C)$ of the complex bilinear form defined by the charge conjugation matrix $C$.

A bilinear form is called admissible if it has a definite symmetry and type. Note that this implies that it is Spino-invariant, since the generators of infinitesimal Lorentz transformations are $\gamma^{\mu \nu}$. The Spin $0_{-}$equivariant vector-valued bilinear form associated to an admissible bilinear form $\beta$ is symmetric if and only if $\sigma(\beta) \tau(\beta)=+1 .^{5}$ We will call such vector-valued forms, and the underlying bilinear forms, super-admissible. ${ }^{6}$ Superbrackets, and the corresponding real, symmetric, Spin ${ }^{-}$-equivariant vector-valued bilinear forms form a vector space, and it was shown in [15] that one can always choose a basis consisting of

\footnotetext{
${ }^{5}$ Note that so far we work with commuting spinors. Spinor fields with anti-commuting components will be introduced later when we construct representations of the supersymmetry algebra on fields.

${ }^{6}$ This is a new terminology not used in [15] which we find convenient.
} 
super-admissible vector-valued bilinear forms. Hence we can restrict ourselves to superadmissible bilinear forms in the following.

So far the spinor module $\mathbf{S}$ has been arbitrary. In the following we focus on the case of the complex spinor module $\mathbb{S}$, that is on supersymmetry algebras where the supercharges form a single Dirac spinor. In odd dimensions, where Dirac spinors are complex irreducible, this covers all the minimal superalgebras, except in signatures where one can obtain a smaller algebra by imposing a Majorana condition. In even dimensions, where Dirac spinors decompose into complex semi-spinors (Weyl spinors) one can also obtain smaller supersymmetry algebras by imposing a chirality condition. General extended superalgebras are obtained by taking multiple copies of real irreducible spinor modules. We will leave these more complicated cases to future work [19, 22], and focus on the case where the supercharges form a single Dirac spinors. Moreover, when determining R-symmetry groups, we will restrict to the case where Dirac spinors are complex irreducible, that is to odd dimensions.

\subsubsection{Standard bilinear forms}

The matrices $A$ and $C$ introduced previously naturally define admissible real bilinear forms on the complex spinor module $\mathbb{S}$. Associated with the matrix $A$ we first have the $\operatorname{Spin}_{0^{-}}$ invariant sesquilinear form

$$
A(\lambda, \chi)=\lambda^{\dagger} A \chi, \quad \lambda, \chi \in \mathbb{S},
$$

which is either Hermitian or anti-Hermitian. By decomposition of $A$ into its real and imaginary part we obtain two admissible real bilinear forms, one symmetric, the other antisymmetric.

We can also define a $\mathrm{Spin}_{0}$-invariant complex bilinear form

$$
C(\lambda, \chi)=\lambda^{T} C \chi
$$

on $\mathbb{S}$ using a charge conjugation matrix $C$. This form has symmetry $\sigma(C)=\sigma$ and type $\tau(C)=\tau$. By decomposition of $C$ into its real and imaginary part we obtain two admissible real bilinear forms, which, depending on $\sigma$, are either both symmetric or both antisymmetric.

\subsubsection{R-symmetry groups}

The R-symmetry group of a supersymmetry algebra is the group of all automorphisms of the superbracket which commute with the Spin representation. Thus for a Poincare Lie superalgebra $\mathfrak{s p}(V)=V+\mathfrak{s} \mathfrak{o}(V)+\mathbf{S}$ this is the group of all automorphisms which act trivially on the even part $V+\mathfrak{s o}(V)$. We first define the Schur algebra $\mathcal{C}(\mathbf{S})$, which consists of all endomorphisms of $\mathbf{S}$ which are invariant under the infinitesimal action of the Spin group:

$$
\mathcal{C}(\mathbf{S})=\{Z \in \operatorname{End}(\mathbf{S}) \mid[Z, \mathfrak{s o}(V)]=0\} .
$$

The invertible elements of the Schur algebra form the Schur group $\mathcal{C}(\mathbf{S})^{*}$. The R-symmetry group can then be defined as the subgroup of the Schur group which leaves the vector-valued 
bilinear form $\beta\left(\gamma^{\mu} \cdot, \cdot\right)$ defining the superbracket invariant:

$$
G_{R}=\left\{Z \in \mathcal{C}(\mathbf{S})^{*} \mid \beta\left(\gamma^{\mu} Z \cdot, Z \cdot\right)=\beta\left(\gamma^{\mu} \cdot, \cdot\right)\right\} .
$$

\subsection{Doubled spinors}

We now introduce doubled spinors which will allow us to define complex supersymmetry algebras in terms of complex vector-valued bilinear forms. We take the spinor module $\mathbf{S}$ to be the complex spinor module $\mathbb{S}$, deferring the general case to a separate publication [19]. We also assume that $\mathbb{S}$ is complex irreducible, which is true in odd dimensions. We do not impose that $\mathbb{S}$ is irreducible as a real representation, that is, in signatures where Majorana spinors are possible one needs to analyze separately whether one can define a smaller supersymmetry algebra. While we show that this does not happen in five dimensions, the general case is left to [19].

Taking the spinor module to be $\mathbb{S}$, the doubled spinor module is $\mathbb{S} \oplus \mathbb{S}$. Since these are two copies of the same $\operatorname{Spin}_{0}(t, s)$ representation we can write this space as a complex tensor product between $\mathbb{S}$ and an internal 'multiplicity space' $\mathbb{C}^{2}$ :

$$
\underline{\lambda}=\left(\lambda^{i}\right)_{i=1,2}=\left(\lambda^{1}, \lambda^{2}\right) \in \mathbb{S} \oplus \mathbb{S} \simeq \mathbb{S} \otimes \mathbb{C}^{2} \ni\left(\lambda_{\alpha}^{i}\right),
$$

where $\alpha=1 \ldots \operatorname{dim} \mathbb{S}$. This product form is convenient since now the Spin group only acts on the first factor, while, as we will show, the R-symmetry group only acts on the second factor. Since $\mathbb{S}$ is a complex $\operatorname{Spin}_{0}(t, s)$ module it automatically carries a representation of the complex Spin group. By equipping $\mathbb{S} \otimes \mathbb{C}^{2}$ with a super-admissible complex vectorvalued bilinear form, we therefore obtain a complex supersymmetry algebra. To pick a real form for a given signature $(t, s)$, we need to choose a $\operatorname{Spin}_{0}(\mathrm{t}, \mathrm{s})$ invariant real structure $\rho$ on $\mathbb{S} \otimes \mathbb{C}^{2}$ and restrict the complex vector-valued bilinear form to the real points $\left(\mathbb{S} \otimes \mathbb{C}^{2}\right)^{\rho}$ with respect to $\rho$. This way we obtain a super-admissible real vector-valued bilinear form on $\left(\mathbb{S} \otimes \mathbb{C}^{2}\right)^{\rho} \simeq \mathbb{S}$. We now explain this construction in detail.

On any complex spinor module $\mathbb{S}$ there exists at least one admissible complex bilinear form, the one defined by the charge conjugation matrix $C$. This bilinear form can be used to define a super-admissible complex vector-valued bilinear form on $\mathbb{S} \otimes \mathbb{C}^{2}$. However, since there might be other admissible complex bilinear forms on $\mathbb{S}$, we use the generic notation

$$
\beta(\lambda, \chi)=\lambda^{T} Q_{\beta} \chi, \quad \lambda, \chi \in \mathbb{C}
$$

in the following. Given any admissible bilinear form $\beta$ on $\mathbb{S}$, we obtain admissible complex bilinear forms on $\mathbb{S} \otimes \mathbb{C}^{2}$ by tensoring $\beta$ with any non-degenerate symmetric or antisymmetric complex bilinear form

$$
M: \mathbb{C}^{2} \ni(w, z) \mapsto M(w, z)=w^{i} z^{j} M_{j i}
$$

on $\mathbb{C}^{2}$. The resulting bilinear form $b=b_{\beta, M}=\beta \otimes M$,

$$
(\beta \otimes M)(\underline{\lambda}, \underline{\chi})=\left(\lambda^{i}\right)^{T} Q_{\beta} \chi^{j} M_{j i}
$$

has symmetry $\sigma\left(b_{\beta, M}\right)=\sigma(\beta) \sigma(M)$ and type $\tau\left(b_{\beta, M}\right)=\tau(\beta)$. 
To obtain a complex supersymmetry algebra with associated spinor module $\mathbb{S} \otimes \mathbb{C}^{2}$, we require that the complex vector-valued billinear form $b_{\beta, M}$ is super-admissible, that is the associated vector-valued form must be symmetric: $\sigma\left(b_{\beta, M}\right) \tau\left(b_{\beta, M}\right)=+1$. Given a bilinear form $\beta$ with $\sigma(\beta) \tau(\beta)=1$, one can therefore use any symmetric bilinear form on $\mathbb{C}^{2}$, while for $\sigma(\beta) \tau(\beta)=-1$ we can use any antisymmetric bilinear form.

Since we want to determine the R-symmetry groups of the real supersymmetry algebas later, it is useful to define complex versions of the Schur algebra and of the R-symmetry group. The Schur algebra $\mathcal{C}\left(\mathbb{S} \otimes \mathbb{C}^{2}\right)$ is defined as the subalgebra of $\operatorname{End}_{\mathbb{C}}\left(\mathbb{S} \otimes \mathbb{C}^{2}\right)$ which commutes with the $\mathrm{Spin}_{0}$-representation. The invertible elements of the Schur algebra form the Schur group $\mathcal{C}^{*}(\mathbb{S}) \subset \operatorname{Aut}_{\mathbb{C}}\left(\mathbb{S} \otimes \mathbb{C}^{2}\right)$. Since we assume that $\mathbb{S}$ is irreducible as a complex

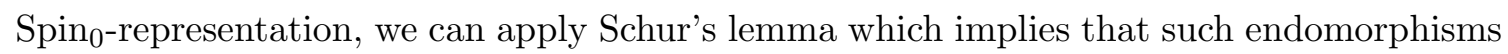
must act on $\mathbb{S}$ as a (non-zero) complex multiple of the unit $\mathbb{1}$. This implies that $\mathcal{C}^{*}\left(\mathbb{S} \otimes \mathbb{C}^{2}\right) \simeq$ $\mathrm{GL}\left(\mathbb{C}^{2}\right) \simeq \mathrm{GL}(2, \mathbb{C})$. We define the complex form of the R-symmetry group to be the subgroup of $\mathcal{C}^{*}\left(\mathbb{S} \otimes \mathbb{C}^{2}\right)$ which leaves the complex vector-valued bilinear form $(\beta \otimes M)\left(\gamma^{\mu} \cdot, \cdot\right)$ invariant. Since $M$ is either a symmetric or an antisymmetric non-degenerate bilinear form, there are only two cases:

$$
G_{R}^{\mathbb{C}} \simeq \begin{cases}\mathrm{Sp}(2, \mathbb{C}), & \text { if } M \text { is antisymmetric } \\ O(2, \mathbb{C}), & \text { if } M \text { is symmetric }\end{cases}
$$

The R-symmetry group of the real theory will then be a real form of $G_{R}^{\mathbb{C}}$. For $G_{R}^{\mathbb{C}}=$ $\mathrm{Sp}(2, \mathbb{C})$ the real R-symmetry group is locally isomorphic to $\mathrm{SU}(2)$ or to $\mathrm{SU}(1,1)$, while for $G_{R}^{\mathbb{C}}=O(2, \mathbb{C})$ it is locally isomorphic to $\mathrm{SO}(2)$ or to $\mathrm{SO}(1,1)$.

Note that in order to apply Schur's lemma, we need to assume that $\mathbb{S}$ is complex irreducible. If $\mathbb{S}$ is complex reducible, as it happens in even dimensions, then the $\mathrm{R}$ symmetry group will be extended by transformations that act non-trivially on the factor $\mathbb{S}$ of $\mathbb{S} \otimes \mathbb{C}^{2}$. See for example [6].

\subsection{Reality conditions}

We now show how to recover Dirac spinors from doubled spinors by imposing a $\operatorname{Spin}_{0^{-}}$ invariant reality condition. The possible reality conditions depend on whether $\mathbb{S}$ carries a real or quaternionic structure.

\subsubsection{Symplectic Majorana spinors}

We start with the case $B B^{*}=-11$, where $\mathbb{S}$ carries a $\operatorname{Spin}_{0}$-invariant quaternionic structure $j_{\mathbb{S}}^{(\alpha)}$. To obtain a $\operatorname{Spin}_{0}$-invariant real structure on $\mathbb{S} \otimes \mathbb{C}^{2}$, we can take the product with any quaternionic structure $j_{\mathbb{C}^{2}}$ on $\mathbb{C}^{2}$. For concreteness, we take the standard quaternionic structure on $\mathbb{C}^{2}$,

$$
j_{\mathbb{C}^{2}}\left[\left(\begin{array}{c}
z_{1} \\
z_{2}
\end{array}\right)\right]=\left(\begin{array}{c}
-z_{2}^{*} \\
z_{1}^{*}
\end{array}\right) .
$$

The quaternionic structure on $\mathbb{S}$ is

$$
j_{\mathbb{S}}(\lambda)=\alpha^{*} B^{*} \lambda^{*}
$$


The resulting real structure $\rho=j_{\mathbb{S}} \otimes j_{\mathbb{C}^{2}}$ is

$$
\rho\left[\left(\begin{array}{c}
\lambda^{1} \\
\lambda^{2}
\end{array}\right)\right]=\left(\begin{array}{c}
-j_{\mathbb{S}} \lambda^{2} \\
j_{\mathbb{S}} \lambda^{1}
\end{array}\right)=\left(\begin{array}{c}
\alpha^{*} B^{*}\left(\lambda^{i}\right)^{*} \varepsilon_{i 1} \\
\alpha^{*} B^{*}\left(\lambda^{i}\right)^{*} \varepsilon_{i 2}
\end{array}\right),
$$

where

$$
\varepsilon=\left(\begin{array}{cc}
0 & 1 \\
-1 & 0
\end{array}\right) .
$$

Symplectic Majorana spinors are by definition those doubled spinor which are real in the sense that they are invariant under $\rho$ :

$$
\rho(\lambda)=\lambda \Leftrightarrow \lambda^{2}=j_{\mathbb{S}} \lambda^{1} \Leftrightarrow\left(\lambda^{i}\right)^{*}=\alpha B \lambda^{j} \varepsilon_{j i}
$$

Since we can identify the subspace $\mathbb{S}_{\mathrm{SM}}=\left(\mathbb{S} \otimes \mathbb{C}^{2}\right)^{\rho} \subset \mathbb{S} \otimes \mathbb{C}^{2} \simeq \mathbb{S} \oplus \mathbb{S}$ of real doubled spinors with the graph of $j_{\mathbb{S}}$ on $\mathbb{S}$, we obtain an isomorphism between Dirac spinors and symplectic Majorana spinors

$$
\mathbb{S} \ni \lambda^{1} \mapsto\left(\lambda^{1}, j_{\mathbb{S}}\left(\lambda^{1}\right)\right) \in \mathbb{S}_{\mathrm{SM}} \subset \mathbb{S} \otimes \mathbb{C}^{2}
$$

This is an isomorphism of real Clifford modules and hence of real Spin modules [6]. The elements $\phi \in \mathrm{GL}(2, \mathbb{C}) \simeq \mathcal{C}^{*}\left(\mathbb{S} \otimes \mathbb{C}^{2}\right)$ of the Schur group which commute with the reality condition form a subgroup. Evaluating the condition

$$
j_{\mathbb{C}^{2}} \varphi(\lambda)=\varphi j_{\mathbb{C}^{2}}(\lambda) \text {, where } j_{\mathbb{C}^{2}}\left(\lambda^{i}\right)=\left(\lambda^{j}\right)^{*} \varepsilon_{j i} .
$$

we find

$$
\varphi=\left(\begin{array}{cc}
u & v \\
-v^{*} & u^{*}
\end{array}\right) \in \mathrm{GL}(1, \mathbb{H}) \subset \mathrm{GL}(2, \mathbb{C}),
$$

where $\mathrm{GL}(1, \mathbb{H})=\mathbb{H}^{*}$ is the group of invertible quaternions. The corresponding Rsymmetry will be worked out below.

\subsubsection{Real doubled spinors defined by a Majorana condition}

In signatures where $B^{*} B=1$, one can define Majorana spinors and possibly obtain a supersymmetry algebra that is smaller than the one based on Dirac spinors. But we can also use the Majorana condition to describe Dirac spinors in terms of real doubled spinors. This time the reality condition imposed on doubled spinors is the product of the $\mathrm{Spin}_{0^{-}}$ invariant real structure $\rho_{\mathbb{S}}$ on $\mathbb{S}$ defined by the Majorana condition, and a real structure on $\mathbb{C}^{2}$. For later convenience we choose

$$
\rho_{\mathbb{C}^{2}}\left[\left(\begin{array}{c}
z_{1} \\
z_{2}
\end{array}\right)\right]=\left(\begin{array}{l}
z_{2}^{*} \\
z_{1}^{*}
\end{array}\right) .
$$

The real structure on $\mathbb{S}$ is

$$
\rho_{\mathbb{S}}(\lambda)=\alpha^{*} B^{*} \lambda^{*}
$$


The resulting real structure on $\mathbb{S} \otimes \mathbb{C}^{2}$ is

$$
\rho\left[\left(\begin{array}{c}
\lambda^{1} \\
\lambda^{2}
\end{array}\right)\right]=\left(\begin{array}{c}
\rho_{\mathbb{S}} \lambda^{2} \\
\rho_{\mathbb{S}} \lambda^{1}
\end{array}\right)=\left(\begin{array}{c}
\alpha^{*} B^{*}\left(\lambda^{i}\right)^{*} \eta_{i 1} \\
\alpha^{*} B^{*}\left(\lambda^{i}\right)^{*} \eta_{i 2}
\end{array}\right),
$$

where

$$
\left(\eta_{i j}\right)=\left(\begin{array}{ll}
0 & 1 \\
1 & 0
\end{array}\right) .
$$

Real doubled spinors are those doubled spinors which are invariant under $\rho$ :

$$
\rho(\lambda)=\lambda \Leftrightarrow \lambda^{2}=\rho_{\mathbb{S}} \lambda^{1} \Leftrightarrow\left(\lambda^{i}\right)^{*}=\alpha B \lambda^{j} \eta_{j i}
$$

Similarly to the symplectic Majorana case, we have an isomorphism between Dirac spinors and real doubled spinors using that the real doubled spinors lie on the graph of the real structure:

$$
\mathbb{S} \ni \lambda^{1} \mapsto\left(\lambda^{1}, \rho_{\mathbb{S}}\left(\lambda^{1}\right)\right) \in \mathbb{S}_{D M} \subset \mathbb{S} \otimes \mathbb{C}^{2} \simeq \mathbb{S} \oplus \mathbb{S} .
$$

To find the Schur group associated with the real theory we need to identify those elements $\varphi \in \mathrm{GL}(2, \mathbb{C}) \simeq \mathcal{C}^{*}\left(\mathbb{S} \otimes \mathbb{C}^{2}\right)$ which commute with the real structure $\rho_{\mathbb{C}^{2}}$. The condition is

$$
\rho_{\mathbb{C}^{2}} \varphi(\lambda)=\varphi \rho_{\mathbb{C}^{2}}(\lambda), \text { where } \rho_{\mathbb{C}^{2}}\left(\lambda^{i}\right)=\left(\lambda^{j}\right)^{*} \eta_{j i} .
$$

which implies

$$
\varphi=\left(\begin{array}{cc}
u & v \\
v^{*} & u^{*}
\end{array}\right) \in \mathrm{GL}\left(1, \mathbb{H}^{\prime}\right) \subset \mathrm{GL}(2, \mathbb{C}),
$$

where $\mathrm{GL}\left(1, \mathbb{H}^{\prime}\right) \simeq \mathrm{GL}(2, \mathbb{R}) \subset \mathrm{GL}(2, \mathbb{C})$ is the group of invertible para-quaternions. ${ }^{7}$

\subsection{Determination of R-symmetry groups}

We have seen that the R-symmetry group of the complex theory is either isomorphic to $O(2, \mathbb{C})$ or to $\operatorname{Sp}(2, \mathbb{C})=\operatorname{SL}(2, \mathbb{C})$, depending on whether the complex bilinear form we choose on $\mathbb{C}^{2}$ is symmetric or antisymmetric. The possible real R-symmetry groups are those subgroups which commute with the reality condition we impose. Therefore the real R-symmetry groups are real forms of the complex R-symmetry groups. For semi-simple groups, such as $\operatorname{Sp}(2, \mathbb{C})$, this implies that they can be obtained as fixed points under an involutive automorphism, see for example [23] for background. The group $O(2, \mathbb{C})$ is abelian, and not semi-simple. Since there are only two connected real one-dimensional Lie groups, $\mathrm{SO}(2)$ and $\mathbb{R} \simeq \mathrm{SO}_{0}(1,1)$, we know that these are the connected components of the real R-symmetry groups. We will show that both the compact and the non-compact form appear, and determine the group globally for the standard involutions.

Let us show explicitly how imposing a reality condition on doubled spinors is related to involutive automorphisms of $G_{R}^{\mathbb{C}}$. The real R-symmetry group is defined by

$$
G_{R}=\left\{\varphi \in G_{R}^{\mathbb{C}} \mid \rho \varphi=\varphi \rho\right\} \subset G_{R}^{\mathbb{C}} \simeq \operatorname{GL}(2, \mathbb{C}),
$$

\footnotetext{
${ }^{7}$ See appendix B for the relevant fact about para-quaternions.
} 
where $\rho$ is either a real structure $\rho_{\mathbb{C}^{2}}$ or a quaternionic structure $j_{\mathbb{C}^{2}}$ on $\mathbb{C}^{2}$. Its action takes the form

$$
\rho\left(z^{i}\right)=z^{j *} N_{j i}, \quad i, j=1,2
$$

where $N=\left(N_{j i}\right)$ is a real invertible matrix which is either symmetric or antisymmetric, and satisfies $N^{2}= \pm \mathbb{1}$. The choices corresponding to the two types of real doubled spinors introduced earlier are $\eta_{j i}$ and $\varepsilon_{j i}$. We parametrize the action of a complex R-symmetry transformation $\varphi \in G_{R}^{\mathbb{C}}$ as

$$
\varphi\left(z^{i}\right)=A_{j}^{i} z^{j}
$$

where $A=\left(A^{i}{ }_{j}\right) \in \mathrm{GL}(2, \mathbb{C})$. Evaluating the condition $\rho \varphi=\varphi \rho$ we find, taking into account that $N^{T}= \pm N$,

$$
A=N A^{*} N^{-1}
$$

This is precisely the action of an involutive automorphism acting by complex conjugation $K$ composed with conjugation by the matrix $N$ :

$$
A \mapsto N \circ K(A)=N K(A) N^{-1}
$$

Since we would like to know the R-symmetry groups for all possible types of reality conditions, we first need to review the relevant facts about real forms of complex Lie groups. For a connected simple complex Lie group, all real forms can be obtained as fixed points of involutive automorphisms. Up to conjugation with inner automorphisms, there are at most three types of such automorphisms: complex conjugation $K$, and the conjugation by matrices of the form

$$
I_{p, q}=\left(\begin{array}{cc}
\mathbb{1}_{p} & 0 \\
0 & -\mathbb{1}_{q}
\end{array}\right), \quad J_{p, p}=\left(\begin{array}{cc}
0 & \mathbb{1}_{p} \\
-\mathbb{1}_{p} & 0
\end{array}\right)
$$

For the connected simple group $G_{R}^{\mathbb{C}}=\operatorname{Sp}(2, \mathbb{C})=\operatorname{SL}(2, \mathbb{C})$, the three basic involutions are $K, I_{1,1}$ and $J_{1,1}=\varepsilon$. We find it convenient to replace $I_{1,1}$ by the equivalent matrix $\eta$, and to use $K, \eta \circ K, \varepsilon \circ K$ as the independent involutions. The group $G_{R}^{\mathbb{C}}=O(2, \mathbb{C})$ has two connected components and is abelian rather than simple. Since there are only two connected one-dimensional Lie groups, $\mathrm{SO}(2) \simeq \mathrm{U}(1)$ and $\mathrm{SO}_{0}(1,1) \simeq \mathbb{R}$ the subgroup of $O(2, \mathbb{C})$ consistent with the reality condition must be locally isomorphic to one of them.

To determine the real R-symmetry groups, we need to solve the equation

$$
A^{*}=N^{-1} A N
$$

for $A \in \operatorname{Sp}(2, \mathbb{C}), O(2, \mathbb{C})$ and $N=\mathbb{1}, \eta, \varepsilon$. Working infinitesimally, $A=\exp (\epsilon a)=\mathbb{1}+$ $\epsilon a+O\left(\epsilon^{2}\right)$, we see that the Lie algebra element $a$ must satisfy the same equation as the group element,

$$
a^{*}=N^{-1} a N .
$$


We start with $A \in \operatorname{Sp}(2, \mathbb{C})$, where

$$
a=\left(\begin{array}{cc}
\alpha & \beta \\
\gamma & -\alpha
\end{array}\right), \quad \alpha, \beta, \gamma \in \mathbb{C}
$$

- $N=\mathbb{1}$. In this case $a$ and $A$ must be real, which implies $G_{R}=\operatorname{Sp}(2, \mathbb{R}) \simeq \mathrm{SU}(1,1)$.

- $N=\eta$. Equation (2.11) implies $\alpha^{*}=-\alpha, \beta=\gamma^{*}$. Writing $\alpha=\alpha_{1}+i \alpha_{2}$, etc. we obtain

$$
a=\left(\begin{array}{cc}
i \alpha_{2} & \beta_{1}+i \beta_{2} \\
\beta_{1}-i \beta_{2} & -i \alpha_{2}
\end{array}\right) .
$$

The Lie algebra spanned by these matrices has two non-compact generators, and therefore is isomorphic to $\mathfrak{s u}(1,1)$. By exponentiation we obtain $G_{R} \simeq \mathrm{SU}(1,1)$.

- $N=\varepsilon$. Equation (2.11) implies $\alpha^{*}=-\alpha, \beta=-\gamma^{*}$. Therefore

$$
a=\left(\begin{array}{cc}
i \alpha_{2} & \beta_{1}+i \beta_{2} \\
-\beta_{1}+i \beta_{2} & -i \alpha_{2}
\end{array}\right)
$$

The Lie algebra spanned by these matrices has three compact generators, and is therefore isomorphic to $\mathfrak{s u}(2)$. By exponentiation we obtain $G_{R} \simeq \mathrm{SU}(2)$.

We remark that the R-symmetry groups $\mathrm{SU}(2)$ and $\mathrm{SU}(1,1)$ obtained for $N=\varepsilon, \eta$ have a natural interpretation in terms of the quaternionic and para-quaternionic structure on the complex spinor module $\mathbb{S}$. For $N=\varepsilon$ the real Schur group was found to be $\operatorname{GL}(1, \mathbb{H}) \subset$ $\mathrm{GL}(2, \mathbb{C})$ and we can obtain the real R-symmetry group by intersecting this with the complex R-symmetry group. This reveals that the R-symmetry group

$$
\mathrm{GL}(1, \mathbb{H}) \cap \mathrm{Sp}(2, \mathbb{C})=\mathrm{U}(1, \mathbb{H})=\mathrm{SU}(2)
$$

is the group of unit norm quaternions, which rotates the $\mathrm{Spin}_{0}$-invariant quaternionic structures $I, J, K$ on $\mathbb{S}$.

Similarly, for $N=\eta$ the real Schur group was found to be $\mathrm{GL}\left(1, \mathbb{H}^{\prime}\right) \subset \mathrm{GL}(2, \mathbb{C})$. The real R-symmetry group obtained by intersecting with the complex R-symmetry group,

$$
\mathrm{GL}\left(1, \mathbb{H}^{\prime}\right) \cap \mathrm{Sp}(2, \mathbb{C})=\mathrm{U}\left(1, \mathbb{H}^{\prime}\right)=\mathrm{SU}(1,1)
$$

is the group of unit norm para-quaternions, which rotates the Spin-invariant paraquaternionic structure $I, J, K$ on $\mathbb{S}$. 
We now turn to $G_{R}^{\mathbb{C}}=O(2, \mathbb{C})$. An element of the complex Lie algebra $\mathfrak{o}(2, \mathbb{C})$ is an antisymmetric complex two-by-two matrix

$$
\left(\begin{array}{cc}
0 & \alpha \\
-\alpha & 0
\end{array}\right), \quad \alpha \in \mathbb{C} .
$$

- $N=\mathbb{1}$. In this case $a$ and $A$ must be real and we obtain $G_{R}=O(2)$.

- $N=\eta$. In this case (2.11) implies $\alpha^{*}=-\alpha$, so that

$$
a=\left(\begin{array}{cc}
0 & i \alpha_{2} \\
-i \alpha_{2} & 0
\end{array}\right), \quad \alpha_{2} \in \mathbb{R} .
$$

This generates a non-compact group, therefore $G_{R} \supset \mathrm{SO}(1,1)_{0} \simeq \mathbb{R}$.

Since $G_{R}^{\mathbb{C}}=O(2, \mathbb{C})$ has two connected components, while the largest possible real R-symmetry group $O(1,1)$ has four connected components, we need additional work to determine the group globally. We use the parametrization

$$
\left(\begin{array}{cc}
\cos z & \sin z \\
-\sin z & \cos z
\end{array}\right) \in \mathrm{SO}(2, \mathbb{C}), \quad\left(\begin{array}{cc}
\cos z & \sin z \\
\sin z & -\cos z
\end{array}\right) \in O(2, \mathbb{C}) \backslash \mathrm{SO}(2, \mathbb{C}) .
$$

For $A \in \mathrm{SO}(2, \mathbb{C})$ equation (2.10) implies

$$
(\cos z)^{*}=\cos z, \quad(\sin z)^{*}=-\sin z .
$$

This has two solutions for $z$,

$$
z=i \chi, \quad z=\pi+i \chi, \quad \chi \in \mathbb{R} .
$$

The resulting matrices $A$ take the form

$$
A= \pm\left(\begin{array}{cc}
\cosh \chi & i \sinh \chi \\
-i \sinh \chi & \cosh \chi
\end{array}\right) \simeq \pm\left(\begin{array}{cc}
\cosh \chi \sinh \chi \\
\sinh \chi & \cosh \chi
\end{array}\right), \quad \chi \in \mathbb{R},
$$

where we made an equivalence transformation corresponding to the map $\left(z^{1}, z^{2}\right) \mapsto$ $\left(z^{1}, i z^{2}\right)$ in the second step. After this transformation $A$ takes the standard form of an $\mathrm{SO}(1,1)$ matrix, and we see that the group we obtain by imposing the reality condition on $\mathrm{SO}(2, \mathbb{C})$ is isomorphic to $\mathrm{SO}(1,1)$.

For $A \in O(2, \mathbb{C}) \backslash \mathrm{SO}(2, \mathbb{C})$, equation (2.10) implies

$$
(\cos z)^{*}=-\cos z, \quad(\sin z)^{*}=\sin z .
$$

This again has two solutions for $z$ :

$$
z=\frac{\pi}{2}+i \chi, \quad z=\frac{3 \pi}{2}+i \chi, \quad \chi \in \mathbb{R} .
$$

The corresponding matrices $A$ take the form

$$
A= \pm\left(\begin{array}{cc}
-i \sinh \chi & \cosh \chi \\
\cosh \chi & i \sinh \chi
\end{array}\right)
$$

These matrices have determinant -1 and extend the R-symmetry group to $O(1,1)$. 
- $N=\varepsilon$. At the infinitesmal level equation (2.11) implies $\alpha=\alpha^{*}$, so that

$$
a=\left(\begin{array}{cc}
0 & \alpha_{1} \\
-\alpha_{1} & 0
\end{array}\right), \quad \alpha_{1} \in \mathbb{R} .
$$

This generates a compact group, so $G_{R} \supset \mathrm{SO}(2)$. To decide whether the group is $O(2)$ or $\mathrm{SO}(2)$, we turn to equation $(2.10)$. For $A \in \mathrm{SO}(2, \mathbb{C})$ we obtain:

$$
(\cos z)^{*}=\cos (z), \quad(\sin z)^{*}=\sin (z)
$$

so that

$$
A=\left(\begin{array}{cc}
\cos \phi & \sin \phi \\
-\sin \phi & \cos \phi
\end{array}\right)
$$

as it must, given the result for the Lie algebra. For $A \in O(2, \mathbb{C}) \backslash \mathrm{SO}(2, \mathbb{C})$ we obtain

$$
(\cos z)^{*}=-\cos z, \quad(\sin z)^{*}=-\sin z .
$$

These two equations have no common solution, therefore the R-symmetry group is $G_{R}=\mathrm{SO}(2)$.

We note that the differences between the R-symmetry groups $O(2), \mathrm{SO}(2), O(1,1)$ can be characterised in terms of their action on $\mathbb{R}^{2} \subset \mathbb{C}^{2}$. While on $\mathbb{C}^{2}$ the signature of a complex bilinear form is not an invariant, the signature of a real bilinear form on $\mathbb{R}^{2}$ is an invariant, which distinguishes $O(2)$ and $O(1,1)$. Moreover, on $\mathbb{R}^{2}$ we can choose an orientation, or a complex structure, which is preserved by $\mathrm{SO}(2) \subset O(2)$, while the full group $O(2)$ also contains transformations which reverse orientation and complex structure. Similarly, $\mathbb{R}^{2}$ can be equipped by a para-complex structure, which is preserved by $\mathrm{SO}(1,1) \subset O(1,1)$ only.

This completes the determination of the R-symmetry groups that can appear in supersymmetry algebras based a single Dirac spinor of supercharges in odd dimensions. We now specialize to five dimensions where we will show explicitly how to relate superbrackets on $\mathbb{S}$ and $\mathbb{S} \otimes \mathbb{C}^{2}$ to one another.

\section{Minimal supersymmetry in five dimensions}

We first collect a few useful facts and relations valid in five dimensions. The complex spinor module $\mathbb{S}$ is irreducible as a complex module. We will see that for some signatures Majorana spinors exist, which means that $\mathbb{S}$ is reducible as a real module, and that smaller, $\mathcal{N}=1$ superalgebras might exist. However, as we show at the end of this section these superalgebras are trivial in the sense that the supercharges simply anti-commute. From the tables in [15] we can read off that there the vector space of symmetric $\operatorname{Spin}_{0}$-equivariant vector-valued bilinear forms is one-dimensional. In other words, for all signatures the superbracket is unique up to rescaling. We will provide explicit realizations of the superbrackets both in terms of Dirac spinors and in terms of doubled Dirac spinors. 


\begin{tabular}{|l|ll|}
\hline & $\operatorname{Re}[A]$ & $\operatorname{Im}[A]$ \\
\hline$(0,5)$ & $(+,+)$ & $(-,+)$ \\
\hline$(1,4)$ & $(-,-)$ & $(+,-)$ \\
\hline$(2,3)$ & $(-,+)$ & $(+,+)$ \\
\hline$(3,2)$ & $(+,-)$ & $(-,-)$ \\
\hline$(4,1)$ & $(+,+)$ & $(-,+)$ \\
\hline$(5,0)$ & $(-,-)$ & $(+,-)$ \\
\hline
\end{tabular}

Table 1. Invariants, $(\sigma= \pm 1, \tau= \pm 1)$, of Dirac bilinear forms in various signatures.

The charge conjugation matrix $C$ is unique up to equivalence and has symmetry $\sigma=$ $\sigma(C)=-1$ and type $\tau=\tau(C)=1$. It is therefore antisymmetric, $C^{T}=-C$. Since $C$ satisfies $C^{-1}=C=C^{\dagger}$, it is then purely imaginary, $C^{*}=-C$. Since $\tau=1$,

$$
\left(\gamma^{\mu}\right)^{T}=\tau C \gamma^{\mu} C^{-1}=C \gamma^{\mu} C^{-1} .
$$

A straightforward calculation shows that $B=\left(C A^{-1}\right)^{T}$ can be re-written as

$$
B=(-1)^{t+1} C A,
$$

and complex conjugation operates on $\gamma$-matrices by

$$
\left(\gamma^{\mu}\right)^{*}=(-1)^{t} \tau B \gamma^{\mu} B^{-1}=(-1)^{t} B \gamma^{\mu} B^{-1} .
$$

By another straightforward calculation we find

$$
B^{*} B=(-1)^{\left(t^{2}+3 t+2\right) / 2} \mathbb{1}=\left\{\begin{array}{l}
-1, \text { for } t=0,1,4,5, \\
+11, \text { for } t=2,3 .
\end{array}\right.
$$

Thus the spinor module $\mathbb{S}$ has an invariant quaternionic structure for Euclidean and Lorentz signature, but an invariant real structure for signatures with two times. For later use we note that

$$
B^{\dagger} C=(-1)^{\left(t^{2}+3 t+2\right) / 2} A=\left\{\begin{array}{l}
-A \text { for } t=0,1,4,5 \\
A \text { for } t=2,3 .
\end{array}\right.
$$

To define the five-dimensional $\mathcal{N}=2$ superalgebras in terms of Dirac spinors, we use the sesquilinear form

$$
A(\lambda, \chi)=\lambda^{\dagger} A \chi
$$

$A$ is Hermitian for $t=0,3,4$ and anti-Hermitian for $t=1,2,5$. The real and imaginary part of $A$ define two admissible real bilinear forms, whose symmetry is determined by the Hermiticity properties of $A$. The type of $A, \operatorname{Re}(A)$ and $\operatorname{Im}(A)$ is +1 if $A$ is a product of an even number of generators and -1 if $A$ is a product of an odd number of generators. The resulting values for the invariants $\sigma$ and $\tau$ are summarized in table 1 . The superbrackets corresponding to the vector-valued bilinear forms $b_{A}\left(\gamma^{\mu},, \cdot\right)=\operatorname{Re} / \operatorname{Im} A\left(\gamma^{\mu} \cdot, \cdot\right)$ are defined by

$$
\left\{\lambda^{\alpha} Q_{\alpha}, \chi^{\beta} Q_{\beta}\right\}=b_{A}\left(\gamma^{\mu} \lambda, \chi\right) P_{\mu} .
$$




\begin{tabular}{|l|ll|}
\hline & $\operatorname{Re}[C]$ & $\operatorname{Im}[C]$ \\
\hline All signatures & $(-,+)$ & $(-,+)$ \\
\hline
\end{tabular}

Table 2. Invariants, $(\sigma= \pm 1, \tau= \pm 1)$, of the charge conjugation bilinear forms in all five dimensional signatures.

Using our spinor conventions summarized in appendix A, the corresponding anti-commutation relations are:

$$
\left\{Q_{\alpha}, Q_{\beta}\right\}= \begin{cases}\operatorname{Re}\left[\gamma^{\mu} A^{-1}\right]_{\alpha \beta} P_{\mu}, & t=0,1,4,5 \\ \operatorname{Im}\left[\gamma^{\mu} A^{-1}\right]_{\alpha \beta} P_{\mu}, & t=2,3 .\end{cases}
$$

We can also define an admissible complex bilinear form based on the charge conjugation matrix $C$,

$$
C(\lambda, \chi)=\lambda^{T} C \chi
$$

which gives rise to two admissible real bilinear forms, $\operatorname{Re}(C)$ and $\operatorname{Im}(C)$. Since the charge conjugation matrix is unique (up to equivalence) with $\sigma=-1, \tau=1$, these forms are not superadmissible, see table 2. However, we can define a complex superbracket on the doubled spinor module $\mathbb{S} \otimes \mathbb{C}^{2}$ using the admissible bilinear form $b_{C, \varepsilon}=C \otimes \varepsilon$ :

$$
b_{C, \varepsilon}(\lambda, \chi)=(C \otimes \varepsilon)(\lambda, \chi)=\lambda^{T} C \chi=\left(\lambda^{i}\right)^{T} C \chi^{j} \varepsilon_{j i}
$$

and the corresponding vector-valued bracket $b_{C, \varepsilon}\left(\gamma^{\mu} \lambda, \chi\right)$.

\subsection{Real doubled spinors in $(0,5),(1,4),(4,1)$ and $(5,0)$ - using the symplec- tic Majorana condition}

In signatures with no or one time-like dimension, the superbracket on $\mathbb{S}$ is given by the real part of

$$
A\left(\gamma^{\mu} \lambda^{1}, \chi^{1}\right)=(-1)^{t}\left(\lambda^{1}\right)^{\dagger} A \gamma^{\mu} \chi^{1}
$$

where $\lambda^{1}, \chi^{1} \in \mathbb{S}$. Since $B^{*} B=-1$, the complex spinor module $\mathbb{S}$ carries a $\operatorname{Spin}_{0}$-invariant quaternionic structure $j_{\mathbb{C}^{2}}$. Therefore we can define symplectic Majorana spinors using the real structure $\rho=j_{\mathbb{S}} \otimes j_{\mathbb{C}^{2}}$. To evaluate the super-admissible bilinear form $b_{C, \varepsilon}\left(\gamma^{\mu} \cdot, \cdot\right)$ on the space $\mathbb{S}_{\mathrm{SM}}=\left(\mathbb{S} \otimes \mathbb{C}^{2}\right)^{\rho}$ of real doubled spinors, we use the reality condition to eliminate the second components of doubled spinors,

$$
\lambda^{2}=\alpha^{*} B^{*}\left(\lambda^{1}\right)^{*}
$$


A short calculation shows ${ }^{8}$

$$
\begin{aligned}
b_{C, \varepsilon}\left(\gamma^{\mu} \lambda, \chi\right)_{\rho} & =\left[\left(\gamma^{\mu} \lambda^{2}\right)^{T} C \chi^{1}-\left(\gamma^{\mu} \lambda^{1}\right)^{T} C \chi^{2}\right]_{\rho} \\
& =\alpha^{*}\left[\left(\lambda^{1}\right)^{\dagger} B^{\dagger}\left(\gamma^{\mu}\right)^{T} C \chi^{1}-\left(\lambda^{1}\right)^{T}\left(\gamma^{\mu}\right)^{T} C B^{*}\left(\chi^{1}\right)^{*}\right] \\
& =\alpha^{*}\left[(-1)^{\left(t^{2}+3+2\right) / 2}\left(\lambda^{1}\right)^{\dagger} A \gamma^{\mu} \chi^{1}-\left(\left(\lambda^{1}\right)^{\dagger} A \gamma^{\mu} \chi^{1}\right)^{*}\right] \\
& =(-1)^{t} \alpha^{*}\left((-1)^{\left(t^{2}+3 t+2\right) / 2}\left(\left(A \lambda^{1}\right)^{\dagger} \gamma^{\mu} \chi^{1}\right)-\left(\left(A \lambda^{1}\right)^{\dagger} \gamma^{\mu} \chi^{1}\right)^{*}\right) .
\end{aligned}
$$

Here we used (3.1) and (3.2). Since $(-1)^{\left(t^{2}+3 t+2\right) / 2}=-1$ for $t=0,1,4,5$, we find

$$
(C \otimes \varepsilon)_{\text {real }}\left(\gamma^{\mu} \lambda, \chi\right)=-2(-1)^{t} \alpha^{*} \operatorname{Re}(A)\left(\gamma^{\mu} \lambda^{1}, \chi^{1}\right) .
$$

Up to a complex overall factor, the super-admissible vector-valued real bilinear forms on real doubled spinors and on Dirac spinors agree and define the same superalgebra.

We make the conventional choice to rescale the complex bilinear form on $\mathbb{S} \otimes \mathbb{C}^{2}$ by a factor $-\frac{1}{2}$ and to set $\alpha=(-1)^{t}$. Then the restriction of $b\left(\gamma^{\mu} \cdot, \cdot\right)=-\frac{1}{2}(C \otimes \varepsilon)\left(\gamma^{\mu} \cdot, \cdot\right)$ to symplectic Majorana spinors agrees with $\operatorname{Re}(A)\left(\gamma^{\mu} \cdot, \cdot\right)$. Using the conventions summarized in appendix $\mathrm{A}$, the five-dimensional $\mathcal{N}=2$ superalgebra takes the form

$$
\left\{Q_{i \alpha}, Q_{i \beta}\right\}=-\frac{1}{2}\left(\gamma^{\mu} C^{-1}\right)_{\alpha \beta} P_{\mu} \varepsilon_{i j},
$$

where the doubled spinors $Q_{i}=\left(Q_{i \alpha}\right)$ are subject to the reality condition

$$
Q_{i}^{*}=(-1)^{t+1} B \varepsilon^{i j} Q_{j} \Leftrightarrow Q^{i *}=(-1)^{t} B Q^{j} \varepsilon_{j i} .
$$

Since $\mathbb{S}$ is irreducible and the bilinear form $\varepsilon$ on $\mathbb{C}^{2}$ is antisymmetric, the R-symmetry group is

$$
G_{R}=\mathrm{GL}(1, \mathbb{H}) \cap \mathrm{SL}(2, \mathbb{C}) \simeq \mathrm{SU}(2) .
$$

\subsection{Real doubled spinors in $(2,3)$ and $(3,2)$ - using the Majorana condition}

In signatures with two time-like dimensions the superbracket on $\mathbb{S}$ is given by the imaginary part of

$$
A\left(\gamma^{\mu} \lambda^{1}, \chi^{1}\right)=(-1)^{t}\left(\lambda^{1}\right)^{\dagger} A \gamma^{\mu} \chi^{1} .
$$

In these signature $B^{*} B=1$, so that $B$ defines a $\operatorname{Spin}_{0}$-invariant real structure $\rho_{\mathbb{S}}$ on $\mathbb{S}$, which allows us to define Majorana spinors. We show at the end of this section that the restriction of the superbracket to Majorana spinors is trivial and does not define a smaller, $\mathcal{N}=1$ superalgebra with four real supercharges. Here we focus on rewriting Dirac spinors as real doubled spinors. By combining the real structure $\rho_{\mathbb{S}}$ with the real structure $\rho_{\mathbb{C}^{2}}$ on $\mathbb{C}^{2}$ defined by (2.8) we obtain the $\operatorname{Spin}_{0}$-invariant real structure $\rho=\rho_{\mathbb{S}} \otimes \rho_{\mathbb{C}^{2}}$ on doubled spinors. Evaluating the invariant bilinear form $b_{C, \varepsilon}\left(\gamma^{\mu} \cdot, \cdot\right)$ on real doubled spinors, we find

$$
\begin{aligned}
b_{C, \varepsilon}\left(\gamma^{\mu} \lambda, \chi\right)_{\mid \text {real }} & =\alpha^{*}\left(\lambda^{1}\right)^{\dagger} B^{\dagger} C \gamma^{\mu} \chi^{1}+\alpha^{*}(-1)^{t}\left(\left(\lambda^{1}\right)^{\dagger} C B \gamma^{\mu} \chi^{1}\right)^{*} \\
& =\alpha^{*}(-1)^{t(t+1) / 2}\left(A\left(\gamma^{\mu} \lambda^{1}, \chi^{1}\right)-A\left(\gamma^{\mu} \lambda^{1}, \chi^{1}\right)^{*}\right) \\
& =\alpha^{*}(-1)^{t(t+1) / 2} 2 i \operatorname{Im}(A)\left(\gamma^{\mu} \lambda^{1}, \chi^{1}\right),
\end{aligned}
$$

\footnotetext{
${ }^{8}$ From now on we will stop 'underlining' doubled spinors, as it should be clear from context whether $\lambda$ refers to a Dirac spinor or a doubled spinor.
} 


\begin{tabular}{|l|l|}
\hline & Reality Condition \\
\hline$(0,5)$ & $\left(\lambda^{i}\right)^{*}=B \lambda^{j} \varepsilon_{j i}$ \\
\hline$(1,4)$ & $\left(\lambda^{i}\right)^{*}=-B \lambda^{j} \varepsilon_{j i}$ \\
\hline$(2,3)$ & $\left(\lambda^{i}\right)^{*}=i B \lambda^{j} \eta_{i j}$ \\
\hline$(3,2)$ & $\left(\lambda^{i}\right)^{*}=-i B \lambda^{j} \eta_{i j}$ \\
\hline$(4,1)$ & $\left(\lambda^{i}\right)^{*}=B \lambda^{j} \varepsilon_{j i}$ \\
\hline$(5,0)$ & $\left(\lambda^{i}\right)^{*}=-B \lambda^{j} \varepsilon_{j i}$ \\
\hline
\end{tabular}

Table 3. Reality Condition in each signature, $B=\left(C A^{-1}\right)^{T}$ is signature dependent.

where we used (3.1), (3.2) and that $(-1)^{\left(t^{2}+3 t+2\right) / 2}=1$ for $t=2,3$. As for the other signatures, we rescale the bilinear on $\mathbb{S} \otimes \mathbb{C}^{2}$ by a factor $-\frac{1}{2}$, so that

$$
b\left(\gamma^{\mu} \lambda, \chi\right)_{\mid \text {real }}=-i(-1)^{t(t+1) / 2} \alpha^{*} \operatorname{Im}(A)\left(\gamma^{\mu} \lambda^{1}, \chi^{1}\right) .
$$

Then by choosing

$$
\alpha=-i(-1)^{t(t+1) / 2}=\left\{\begin{array}{l}
i \quad \text { for } t=2, \\
-i \text { for } t=3,
\end{array}\right.
$$

the invariant vector-valued bilinear form on $\mathbb{S} \otimes \mathbb{C}^{2}$ agrees with the invariant vector-valued real bilinear form on $\mathbb{S}$ when evaluated on real points. Note that with our conventional choices of $\alpha$, the five-dimensional supersymmetry algebra takes the same form for all signatures when expressed using doubled spinors. The reality conditions which relate doubled spinors to Dirac spinors are summarized in table 3. Since we use the antisymmetric bilinear form $\varepsilon$ on $\mathbb{C}^{2}$, the R-symmetry group is

$$
G_{R}=\mathrm{GL}\left(1, \mathbb{H}^{\prime}\right) \cap \mathrm{Sp}(2, \mathbb{C}) \simeq \mathrm{SU}(1,1) .
$$

\subsection{Reality properties of spinor bilinears and R-group tensors}

In explicit calculations it is convenient to employ a formalism where R-symmetry indices $i, j=1,2$ can be raised and lowered. This is always done using the antisymmetric bilinear form $\varepsilon$ on $\mathbb{C}^{2}$, irrespective of the reality condition. Spinors form R-symmetry doublets, and the rules for raising and lowering indices are

$$
\lambda^{i} \varepsilon_{i j}=\lambda_{j}, \quad \lambda^{i}=\varepsilon^{i j} \lambda_{j},
$$

where $\varepsilon^{i j} \varepsilon_{j k}=-\delta_{k}^{i}$. This rule for raising and lowering indices conforms with the NW-SE convention. Besides spinors, which transform as R-symmetry doublets, the only other field in a five-dimensional vector multiplet that transforms non-trivially under R-symmetry is the auxiliary symmetric tensor field $Y^{i j}$. The rules for raising and lowering indices are

$$
Y^{i j}=\varepsilon^{i k} \varepsilon^{j l} Y_{k l}, \quad Y^{k l} \varepsilon_{k i} \varepsilon_{l j}=Y_{i j} .
$$


Spinors and as well the symmetric tensor $Y^{i j}$ are subject to reality conditions, which involve the indices $i, j$. For spinors we have

$$
\left(\lambda^{i}\right)^{*}=\left\{\begin{array}{l}
\alpha B \lambda^{j} \varepsilon_{j i} \text { for } t=0,1,4,5, \\
\alpha B \lambda^{j} \eta_{j i} \text { for } t=2,3
\end{array} .\right.
$$

For symmetric tensors we then have to take the induced reality conditions

$$
\left(Y^{i j}\right)^{*}= \begin{cases}Y^{k l} \varepsilon_{k i} \varepsilon_{l j}=Y_{i j}, & \text { for } t=0,1,4,5, \\ Y^{k l} \eta_{k i} \eta_{l j}, & \text { for } t=2,3 .\end{cases}
$$

These reality conditions are invariant under the respective R-symmetry groups $\mathrm{SU}(2)$ and $\mathrm{SU}(1,1)$, which act by

$$
Y \rightarrow Y^{\prime}=U Y U^{T}
$$

where

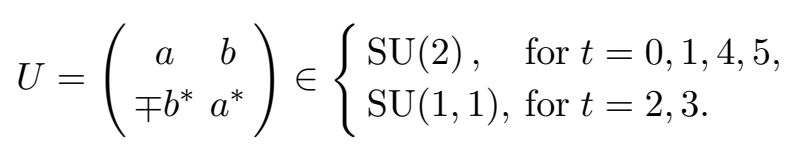

Note that $U$ satisfies

$$
U^{*}=\varepsilon U \varepsilon^{T}, \quad U^{*}=\eta U \eta
$$

respectively. ${ }^{9}$ From this it follows immediately that $Y^{\prime}$ satisfies the same reality condition as $Y$.

Note that for $t=2,3\left(Y^{i j}\right)^{*} \neq Y_{i j}$. The explicit relations between tensor components are:

$$
Y^{11}=Y_{22}, \quad Y^{12}= \pm Y_{12}, \quad Y^{22}=Y_{11}
$$

and

$$
\left(Y^{11}\right)^{*}=Y_{11}, \quad\left(Y^{12}\right)^{*}= \pm Y_{12}, \quad\left(Y^{22}\right)^{*}=Y_{22},
$$

where the plus sign applies for $t=0,1,4,5$ and the minus sign for $t=2,3$. We also note for later use that $Y^{i j} Y_{i j}$ is real for all signatures.

The final ingredient we need is to determine the reality properties of spinor bilinears involving real doubled spinors, as these will appear in the supersymmetry transformations and supersymmetric Lagrangians. For the scalar bilinear, we find

$$
(\bar{\lambda} \chi)^{*}=(-1)^{t} \bar{\lambda} \chi
$$

This formula only depends on $t$ and does not depend on whether $\mathbb{S}$ carries a quaternionic or real structure. The reason is that signs which are sensitive to the type of the reality condition cancel. To see this, write out the expression

$$
\left.(\bar{\lambda} \chi)^{*}=\left(\left(\lambda^{i}\right)^{T} C \chi^{j}\right) \varepsilon_{j i}\right)^{*}
$$

using the reality condition $\left(\lambda^{i}\right)^{*}=\alpha B \lambda^{j} M_{j i}$, where $M_{j i}=\varepsilon_{j i}$ or $M_{j i}=\eta_{j i}$,

$$
(\bar{\lambda} \chi)^{*}=-\alpha^{2}\left(\lambda^{k}\right)^{T} B^{T} C B \chi^{i} M_{k i} M_{l j} \varepsilon^{j i} .
$$

\footnotetext{
${ }^{9}$ These relations show that for both groups the $[2]$ and $[\overline{2}]$ representations are equivalent as real representations.
} 
Using the relations for $A, B, C$ given previously, one finds

$$
B^{T} C B=(-1)^{t+1} C .
$$

We also need

$$
\varepsilon_{k i} \varepsilon_{l j} \varepsilon^{j i}=\varepsilon_{l k}, \quad \eta_{k i} \eta_{l j} \varepsilon^{j i}=-\varepsilon_{l k} .
$$

These relations contain a sign which depends on the type of reality condition we use. However since $\alpha= \pm 1$ for $t=0,1,4,5$, but $\alpha= \pm i$ for $t=2,3$ this sign cancels against $\alpha^{2}= \pm 1$, and we obtain (3.8).

Since

$$
\left(\gamma^{\mu_{1} \cdots \mu_{l}}\right)^{*}=(-1)^{t l} B \gamma^{\mu_{1} \cdots \mu_{l}} B^{-1},
$$

this immediately generalizes to

$$
\left(\bar{\lambda} \gamma^{\mu_{1} \cdots \mu_{l}} \chi\right)^{*}=(-1)^{t(l+1)} \bar{\lambda} \gamma^{\mu_{1} \cdots \mu_{l}} \chi .
$$

Thus for $t=0$ all spinor bilinears are real, while for $t=1$ they alternate between imaginary and real. Note that the vector bilinear, $l=1$, is real for all $t$, as it must, as this is the bilinear which defines the real supersymmetry algebra.

We conclude by noting that all expressions with completely contracted auxiliary indices $i, j$ are $\mathrm{R}$-symmetry invariant: before imposing the reality condition they are manifestly $\operatorname{Sp}(2, \mathbb{C})$-invariant, and the R-symmetry group is precisely the subgroup of $\operatorname{Sp}(2, \mathbb{C})$ which commutes with the reality condition.

\subsection{No $\mathcal{N}=1$ superymmetry algebras in signatures $(2,3)$ and $(3,2)$}

For $t=2,3$, the complex spinor module $\mathbb{S}$ carries a Spin-invariant real structure $J$ defined by the matrix $B$, and we can define Majorana spinors by imposing $\lambda^{*}=\alpha B \lambda$. Thus we might be able to define an $N=1$ superalgebra by restricting the super-admissible bilinear form $b=\operatorname{Im}(A)$ to $J$-invariant spinors. However by explicit computation it is straightforward to verify that the $\operatorname{Spin}_{0}$-invariant endomorphism $J: \lambda \mapsto \alpha^{*} B^{*} \lambda^{*}$ satisfies

$$
b\left(\gamma^{\mu} J \lambda, \chi\right)=-b\left(\gamma^{\mu} \lambda, J \chi\right)
$$

for all signatures, which implies that the bilinear form is identically zero when restricted to $J$-invariant spinors. The corresponding supersymmetry algebra is trivial, that is, supercharges simply anti-commute. Therefore one cannot define an $\mathcal{N}=1$ supersymmetry algebra in five dimensions even in those signatures where Majorana spinors exist. This conclusion can also be reached by analyzing the tables in [15].

\section{Five-dimensional vector multiplets}

We will now derive the off-shell supersymmetry transformations and the general Lagrangians for five-dimensional vector multiplets in arbitrary signature $(t, s)$. Since we can build on the results of [6] for signature $(1,4)$, we proceed as follows. Initially, we work on the doubled spinor module $\mathbb{S} \otimes \mathbb{C}^{2}$ without imposing a reality condition. By allowing arbitrary 
complex coefficients in the supersymmetry transformations and in the Lagrangian known from [6], we obtain all conditions on these parameters which are independent of signature. The resulting transformations and Lagrangian can be viewed as a common complexification of all the five-dimensional theories. In a second step we impose the appropriate reality conditions for each signature, and obtain further signature dependent conditions on the parameters. We will show that consistent solutions exist for all signatures, and are unique up to conventional choices. The transformations and Lagrangians vary from signature to signature only by sign flips or insertion of factors of $\pm i$. Moreover, theories in signatures $(t, s)$ and $(s, t)$ are related by changing the convention for the space-time metric from 'mostly plus' to 'mostly minus', plus taking into account changes in the reality properties of spinor bilinears. Thus we end up with three physically distinct cases: Euclidean theories with signatures $(0,5)$ and $(5,0)$, Lorentzian theories with signatures $(1,4)$ and $(4,1)$ and exotic theories with two time-like directions, with signatures $(2,3)$ and $(3,2)$.

\subsection{Supersymmetry variations}

We have seen that when using doubled spinors the supersymmetry algebra to takes the form

$$
\left\{Q_{\alpha i}, Q_{\alpha j}\right\}=-\frac{1}{2}\left(\gamma^{\mu} C^{-1}\right)_{\alpha \beta} P_{\mu} \varepsilon_{i j} .
$$

To show that this algebra is represented on a collection of space-time fields, we must verify that

$$
\left[\bar{\epsilon}_{(1)} Q, \bar{\epsilon}_{(2)} Q\right] \Phi(x)=-\frac{1}{2}\left(\bar{\epsilon}_{(1)} \gamma^{\mu} C^{-1} \epsilon_{(2)}\right) \partial_{\mu} \Phi(x)
$$

for all fields $\Phi(x)$ belonging to the given supermultiplet. The supersymmetry parameters $\epsilon_{(i)}$ and the fermionic fields $\lambda^{i}$ are anti-commuting spinor fields. ${ }^{10}$ In implementing the supersymmetry algebra, we have chosen that the translation operator $P_{\mu}$ acts as $\partial_{\mu}$ on fields, which is the same convention as in [24]. ${ }^{11}$

The content of a five-dimensional off-shell vector multiplets is known from signature $(1,4)$ :

$$
\left(A^{\mu}, \lambda^{i}, \sigma, Y^{i j}\right), \quad \mu=1,2,3,4,5, \quad i=1,2 .
$$

Apart from the eponymous vector field, $A^{\mu}$, a vector multiplet contains a pair of spinors, $\lambda^{i}$, a scalar field, $\sigma$, and a triplet of auxiliary field combined into a symmetric tensor, $Y^{i j}$. At this point no reality conditions are imposed on $\lambda^{i}$ and $Y^{i j}$, and therefore $A^{\mu}$ and $\sigma$ should be considered as complex fields in order to balance bosonic and fermionic degrees of freedom.

Since we anticipate that the numerical coefficients of terms in the supersymmetry variations will depend on the reality conditions we will impose later, we take the off-shell

\footnotetext{
${ }^{10}$ Note that so far we have been using commuting spinors. Going from commuting to anti-commuting spinors is a 'natural' (functorial) operation and introduces signs in any operation which involves changing the order of spinors.

${ }^{11}$ In the mathematics literature translations are taken to act by $-\partial_{\mu}$ on functions, since this is the infinitesimal action of the one-parameter group $\varphi_{t}: \mathbf{x} \mapsto \mathbf{x}+t \mathbf{e}_{\mu}$ of diffeomorphisms on functions.
} 
supersymmetry transformations derived in $[6]$ for signature $(1,4)$, but replace the numerical pre-factors of all terms by complex coefficients:

$$
\begin{aligned}
\delta A^{\mu} & =\alpha \bar{\epsilon} \gamma^{\mu} \lambda, \quad \delta \sigma=a \bar{\epsilon} \lambda, \quad \delta Y^{i j}=u \bar{\epsilon}^{\left(i \not \partial \lambda^{j}\right),} \\
\delta \lambda^{i} & =\beta \gamma \cdot F \epsilon^{i}+b \not \partial \sigma \epsilon^{i}+y Y^{i j} \epsilon_{j} .
\end{aligned}
$$

By imposing that the transformations satisfy (4.1), and therefore realize the complex supersymmetry algebra, we obtain the following independent relations between the coefficients:

$$
-\frac{1}{2}=-2 a b=4 \alpha \beta=-u y \text {. }
$$

We remark that the calculation leading to these relations is identical to the one done previously in [6], except that we have replaced numerical coefficients by parameters.

The relations (4.4) can be solved for $b, \beta, y$ in terms of $a, \alpha, u$ :

$$
b=\frac{1}{4 a}, \quad \beta=-\frac{1}{8 \alpha}, \quad y=\frac{1}{2 u} .
$$

We now impose reality conditions, proceeding signature by signature. For the scalar $\sigma$ and the vector $A^{\mu}$ this simply means that they are now real-valued. The reality conditions imposed on the spinors $\lambda^{i}, \epsilon^{i}$ were given in (3.6) while those imposed on the auxiliary tensor $Y^{i j}$ were given in (3.7). With regard to the reality properties of spinor bilinears we need to take into account that in a field theory realisation spinors are anticommuting quantities. This leads to extra minus signs when applying operations which change the order of spinors, such as transposition or Hermitian conjugation. Complex conjugation can either be defined to change the order of anticommuting fields or not, and both conventions can be mapped to each other by changing the phase factor in the reality condition $[21,25] .{ }^{12}$ For us it is convenient to keep the reality conditions we fixed in the previous section, and therefore we define the complex conjugation of anti-commuting spinors such that it does not change the order. Then we can use (3.9) to work out the reality properties of the parameters $\alpha, a$ by imposing that $\delta \sigma$ and $\delta A^{\mu}$ are real. Similary, by imposing reality on the variations $\delta Y^{i j}$ and $\delta \lambda^{i}$, we obtain the reality conditions for $u, \beta, b, y$. The resulting conditions are summarized in table 4.

These conditions are consistent with (4.4), because the products $a b, \alpha \beta$, uy are real for all signatures. For signature $(1,4)$ the resulting reality properties of symplectic Majorana spinor bilinears and supersymmetry variations agree with [25] and [6]. For concreteness, we now make explicit choices for $a, \alpha, u$, which then determine $b, \beta, y$ through (4.4). For $(1,4)$ we make a choice which reproduces [6]. For other signatures we then introduce factors $-1, \pm i$, where needed. We summarize the resulting values for the parameters in table 5 .

\subsection{Supersymmetric Lagrangians}

The representations we have found above are off-shell and therefore independent of a choice of field equations or Lagrangian. To specify Lagrangians we proceed in the same way as

\footnotetext{
${ }^{12}$ This phase factor was denoted $\alpha$ previously, but is of course distinct from the parameter $\alpha$ in the supersymmetry transformations. Since by now the reality conditions have been fixed according to table 3 this should not cause any confusion.
} 


\begin{tabular}{|l|l|l|}
\hline Parameter & Real & Imaginary \\
\hline$\alpha$ & $t=0, \ldots 5$ & never \\
\hline$a$ & $t=0,2,4$ & $t=1,3,5$ \\
\hline$\beta$ & $t=0, \ldots, 5$ & never \\
\hline$b$ & $t=0,2,4$ & $t=1,3,5$ \\
\hline$u$ & $t=0,1,4,5$ & $t=2,3$ \\
\hline$y$ & $t=0,1,4,5$ & $t=2,3$ \\
\hline
\end{tabular}

Table 4. Reality conditions for the coefficients of the supersymmetry transformations.

\begin{tabular}{|c|c|c|c|c|c|c|}
\hline & $\alpha$ & $a$ & $u$ & $\beta$ & $b$ & $y$ \\
\hline$(0,5)$ & $\frac{1}{2}$ & $\frac{1}{2}$ & $-\frac{1}{2}$ & $-\frac{1}{4}$ & $\frac{1}{2}$ & -1 \\
\hline$(1,4)$ & $\frac{1}{2}$ & $\frac{i}{2}$ & $-\frac{1}{2}$ & $-\frac{1}{4}$ & $-\frac{i}{2}$ & -1 \\
\hline$(2,3)$ & $\frac{1}{2}$ & $\frac{1}{2}$ & $-\frac{i}{2}$ & $-\frac{1}{4}$ & $\frac{1}{2}$ & $i$ \\
\hline$(3,2)$ & $\frac{1}{2}$ & $\frac{i}{2}$ & $-\frac{i}{2}$ & $-\frac{1}{4}$ & $-\frac{i}{2}$ & $i$ \\
\hline$(4,1)$ & $\frac{1}{2}$ & $\frac{1}{2}$ & $-\frac{1}{2}$ & $-\frac{1}{4}$ & $\frac{1}{2}$ & -1 \\
\hline$(5,0)$ & $\frac{1}{2}$ & $\frac{i}{2}$ & $-\frac{1}{2}$ & $-\frac{1}{4}$ & $-\frac{i}{2}$ & -1 \\
\hline
\end{tabular}

Table 5. Explicit choices for the parameters in the supersymmetry transformations for all signatures.

with the supersymmetry transformations. In [6] the general (two derivative) rigid off-shell Lagrangian for vector multiplets in signature $(1,4)$ was determined. To adapt this result to other signatures, we introduce parameters $s_{F}, s_{\sigma}, s_{\lambda}, s_{Y}, \theta_{1}, \theta_{2}, \theta_{3}$, which are fixed later by imposing reality conditions.

The general form of the Lagrangian is

$$
\begin{aligned}
L= & \left(\frac{s_{F}}{4} F_{\mu \nu}^{I} F^{J \mu \nu}+\frac{s_{\sigma}}{2} \partial_{\mu} \sigma^{I} \partial^{\mu} \sigma^{J}+\frac{s_{\lambda}}{2} \bar{\lambda}^{I} \not \partial \lambda^{J}+s_{Y} Y_{i j}^{I} Y^{J i j}\right) \mathcal{F}_{I J}(\sigma) \\
& +\left(\theta_{1} \varepsilon^{\mu \nu \rho \sigma \tau} A_{\mu}^{I} F_{\nu \rho}^{J} F_{\sigma \tau}^{K}+\theta_{2} \bar{\lambda}^{I} \gamma^{\mu \nu} F_{\mu \nu}^{J} \lambda^{K}+\theta_{3} \bar{\lambda}^{I i} \lambda^{J j} Y_{i j}^{K}\right) \mathcal{F}_{I J K}(\sigma)
\end{aligned}
$$

Vector multiplets are labeled by $I, J=1, \ldots, N$. Initially we do not impose reality conditions, so that all quantities are complex. Note that this Lagrangian is complex-valued, and holomorphic, since it does not involve complex conjugation. As in [19] it does not seem to have a direct physical interpretation ${ }^{13}$ but can be thought of as a 'holomorphic master Lagrangian,' encoding all the possible real forms. Imposing invariance under the supersymmetry transformations (4.3) involves the same computational steps as verifying the supersymmetry invariance of the $(1,4)$ theory in [6]. Therefore the essential structural properties carry over to all signatures. In particular, the scalar field dependent couplings

\footnotetext{
${ }^{13}$ Except possibly in terms of a complexified configuration space which contains the complex saddle points of a Euclidean functional integral, see for example [26, 27].
} 
$\mathcal{F}_{I J}$ and $\mathcal{F}_{I J K}$ can be expressed as derivatives of a Hesse potential $\mathcal{F}(\sigma):^{14}$

$$
\mathcal{F}_{I J}(\sigma)=\frac{\partial}{\partial \sigma^{I}} \frac{\partial}{\partial \sigma^{J}} \mathcal{F}(\sigma), \quad \mathcal{F}_{I J K}(\sigma)=\frac{\partial}{\partial \sigma^{I}} \frac{\partial}{\partial \sigma^{J}} \frac{\partial}{\partial \sigma^{K}} \mathcal{F}(\sigma) .
$$

Supersymmetry requires that a Chern-Simons term is included, unless the theory is noninteracting and thus the Hesse potential quadratic. Gauge invariance of the Chern-Simons term, up to boundary terms, implies that $\mathcal{F}(\sigma)$ must be a polynomial of degree at most three. As noted in [6] the spinor term can be written using a partial derivative, rather than using the covariant derivative with respect to the Levi-Civita connection of the scalar manifold, because the difference is identically zero due to the property $\bar{\lambda}^{i(I \mid} \lambda^{j \mid J)} \varepsilon_{j i}=0$ of spinor bilinears, which holds irrespective of reality conditions.

Invariance of the Lagrangian under the transformations (4.3) imposes the following conditions on the coefficients:

$$
\begin{aligned}
& s_{F} \alpha=-2 s_{\lambda} \beta, \quad s_{\sigma} a=-s_{\lambda} b, \quad 2 s_{Y} u=-s_{\lambda} y, \\
& 3 \theta_{1} \alpha= \pm 2 i^{t} \theta_{2} \beta, \quad 4 \theta_{2} \alpha=-\theta_{3} u, \quad \theta_{2} y=\theta_{3} \beta, \\
& \alpha s_{F}=8 b \theta_{2}, \quad a s_{Y}=y \theta_{3}, \quad a s_{\lambda}=8 \alpha \theta_{2} \text {. }
\end{aligned}
$$

The sign appearing in the relation determining $\theta_{1}$ will be explained below.

The relations (4.7) are consistent but not independent. Using the relations (4.4) we can rearrange the condition imposed on the coefficients $s_{i}$ as follows:

$$
-\frac{1}{2}=-2 \frac{s_{F}}{s_{\lambda}} \alpha^{2}=2 \frac{s_{\sigma}}{s_{\lambda}} a^{2}=2 \frac{s_{Y}}{s_{\lambda}} u^{2}
$$

These relations determine the relative signs of the quadratic terms in the Lagrangian. The coefficients $\theta_{2}, \theta_{3}$ are then determined in terms of any of the $s_{i}$, say $s_{\lambda}$ for concreteness, as:

$$
\theta_{2}=\frac{a}{8 \alpha} s_{\lambda}, \quad \theta_{3}=-\frac{9}{16 u} s_{\lambda} .
$$

Finally, $\theta_{1}$ is determined by

$$
\theta_{1}= \pm \frac{2}{3} i^{t} \theta_{2} \frac{\beta}{\alpha}= \pm \frac{2}{3} i^{t} \frac{a \beta}{8 \alpha^{2}} s_{\lambda}= \pm \frac{1}{24},
$$

with a sign that can be adjusted by making conventional choices. In odd dimensions the volume element $\omega=\gamma_{1} \cdots \gamma_{t+s}$ of the Clifford algebra commutes with the generators $\gamma_{\mu}$ and is therefore proportional to the unit $\mathbb{1}$ on irreducible representations. Since in five dimensions $\omega^{2}=(-1)^{t} \mathbb{1}$, this implies that $\omega= \pm \mathbb{1}$ for even $t$ and $\omega= \pm i \mathbb{1}$ for odd $t$. The choice of a sign, together with the definition of the completely antisymmetric tensor $\varepsilon_{\mu \nu \rho \sigma \tau}$ determines the relative factor in the relation $\gamma_{\mu \nu \rho \sigma \tau} \propto \varepsilon_{\mu \nu \rho \sigma \tau} \mathbb{1}$ for completely antisymmetrized products of five generators. The choices we make are $\omega=\mathbb{1}$ for even $t$, $\omega=-i \mathbb{1}$ for odd $t$ and $\varepsilon_{01234}=+1$ for all signatures. Then

$$
\gamma_{\mu \nu \rho \sigma \tau}=\left\{\begin{array}{c}
\varepsilon_{\mu \nu \rho \sigma \tau} \mathbb{1 1}, t \text { even }, \\
-i \varepsilon_{\mu \nu \rho \sigma \tau} 11, t \text { odd } .
\end{array}\right.
$$

\footnotetext{
${ }^{14}$ As long as we do not impose reality conditions this is a holomorphic Hesse potential for a complexRiemannian metric.
} 
These choices are relevant when relating the $\gamma$ and $4-\gamma$ terms arising from the supersymmetry variations of the Chern Simons term and of the term $\bar{\lambda}^{I} \gamma^{\mu \nu} F_{\mu \nu}^{J} \lambda^{K}$. With our conventions the Lagrangian is supersymmetric for all signatures if $\theta_{1}=+\frac{1}{24}$.

The values of $\alpha^{2}, a^{2}, u^{2}$ depend on the signature, leading to sign flips of the respective terms between signatures. For reference we will now list the Lagrangians and supersymmetry transformations for all signatures.

\subsection{Overview and discussion of supersymmetry variations and Lagrangians by signature}

\subsubsection{Signature $(1,4)$}

In signature $(1,4)$, the relations (4.8) imply: $s_{\sigma}=s_{\lambda}=s_{F}=-s_{Y}= \pm 1$. Thus all physical fields have the same sign in front of their kinetic terms. While the overall sign of the Lagrangian cannot be fixed by imposing invariance under supersymmetry, there is a standard choice in Minkowski signature, namely the choice which makes the kinetic terms of all physical fields positive definite. Therefore we choose

$$
s_{\sigma}=s_{\lambda}=s_{F}=-s_{Y}=-1 .
$$

The remaining coefficients can then be determined from (4.7). We find

$$
\theta_{2}=\frac{i}{8} s_{F}=-\frac{i}{8}, \quad \theta_{3}=-\frac{i}{2} s_{Y}=-\frac{i}{2} .
$$

Finally, as explained above, we have made conventional choices such that $\theta_{1}=\frac{1}{24}$ for all signatures.

Having determined all coefficients, we now summarize the resulting $(1,4)$ signature Lagrangian and supersymmetry transformations:

$$
\begin{aligned}
L_{(1,4)}= & \left(-\frac{1}{4} F_{\mu \nu}^{I} F^{J \mu \nu}-\frac{1}{2} \partial_{\mu} \sigma^{I} \partial^{\mu} \sigma^{J}-\frac{1}{2} \bar{\lambda}^{I} \not \partial \lambda^{J}+Y_{i j}^{I} Y^{i j J}\right) \mathcal{F}_{I J} \\
& +\left(\frac{1}{24} \varepsilon^{\mu \nu \rho \sigma \tau} A_{\mu}^{I} F_{\nu \rho}^{J} F_{\sigma \tau}^{K}-\frac{i}{8} \bar{\lambda}^{I} \gamma^{\mu \nu} F_{\mu \nu}^{J} \lambda^{K}-\frac{i}{2} \bar{\lambda}^{I i} \lambda^{J j} Y_{i j}^{K}\right) \mathcal{F}_{I J K} . \\
\delta A_{\mu}^{I}= & \frac{1}{2} \bar{\epsilon} \gamma_{\mu} \lambda^{I}, \quad \delta \sigma^{I}=\frac{i}{2} \bar{\epsilon} \lambda^{I}, \quad \delta Y^{i j I}=-\frac{1}{2} \bar{\epsilon}^{\left(i \not \partial \lambda^{j}\right) I}, \\
\delta \lambda^{i I}= & -\frac{1}{4} \gamma^{\mu \nu} F_{\mu \nu}^{I} \epsilon^{i}-\frac{i}{2} \not \partial \sigma^{I} \epsilon^{i}-Y^{i j I} \epsilon_{j} .
\end{aligned}
$$

These results agree with [6]. Note that in [6] the Chern-Simons terms appears with the opposite sign, because there the $\varepsilon$-tensor was defined with a relative minus sign, compared to our definition above.

Since the scalar fields are now real, we recover the well known affine special real geometry of five-dimensional vector multiplets, where the scalar metric is a Hessian metric with a cubic polynomial as Hesse potential. This cubic potential needs to be chosen such that the resulting metric is positive definite, a condition that needs to investigated case by case. 


\subsubsection{Signature $(4,1)$}

Next consider signature $(4,1)$, which we interpret as Minkowski signature with a mostly minus convention for the metric. Now (4.8) implies

$$
-s_{\sigma}=s_{\lambda}=s_{F}=-s_{Y}= \pm 1 .
$$

This time the scalar and vector kinetic have different signs, but this only reflects that we now use a different convention for the metric. The standard choice where all physical fields have a positive definite kinetic term is

$$
-s_{\sigma}=s_{\lambda}=s_{F}=-s_{Y}=-1
$$

From this we determine ${ }^{15}$

$$
\theta_{1}=\frac{1}{24}, \quad \theta_{2}=-\frac{1}{8}, \quad \theta_{3}=-\frac{1}{2} .
$$

The resulting Langrangian and supertransformations are:

$$
\begin{aligned}
L_{(4,1)}= & \left(-\frac{1}{4} F_{\mu \nu}^{I} F^{J \mu \nu}+\frac{1}{2} \partial_{\mu} \sigma^{I} \partial^{\mu} \sigma^{J}-\frac{1}{2} \bar{\lambda}^{I} \not \partial \lambda^{J}+Y_{i j}^{I} Y^{i j J}\right) \mathcal{F}_{I J} \\
& +\left(\frac{1}{24} \varepsilon^{\mu \nu \rho \sigma \tau} A_{\mu}^{I} F_{\nu \rho}^{J} F_{\sigma \tau}^{K}-\frac{1}{8} \bar{\lambda}^{I} \gamma^{\mu \nu} F_{\mu \nu}^{J} \lambda^{K}-\frac{1}{2} \bar{\lambda}^{I i} \lambda^{J j} Y_{i j}^{K}\right) \mathcal{F}_{I J K} . \\
\delta A_{\mu}^{I}= & \frac{1}{2} \bar{\epsilon} \gamma_{\mu} \lambda^{I}, \quad \delta \sigma^{I}=\frac{1}{2} \bar{\epsilon} \lambda^{I}, \quad \delta Y^{i j I}=-\frac{1}{2} \bar{\epsilon}^{(i} \not \partial \lambda^{j) I}, \\
\delta \lambda^{i I}= & -\frac{1}{4} \gamma^{\mu \nu} F_{\mu \nu}^{I} \epsilon^{i}+\frac{1}{2} \not \partial \sigma^{I} \epsilon^{i}-Y^{i j I} \epsilon_{j} .
\end{aligned}
$$

When comparing the signatures $(1,4)$ and $(4,1)$ we observe that they differ by factors \pm 1 and $\pm i$, which can be interpreted as resulting from changing from a 'mostly plus' to a 'mosly minus' convention for the metric. For some of the fermionic terms this involves a factor of $i$ due to the signature dependence of the reality properties of spinor bilinears. Interpreting the single distinguished direction as time, both theories are equivalent.

\subsubsection{Signature $(0,5)$}

Now we turn to Euclidean signatures. For signature $(0,5),(4.8)$ implies

$$
-s_{\sigma}=s_{\lambda}=s_{F}=-s_{Y}= \pm 1 .
$$

We observe that the Euclidean action has a relative sign between the scalar and vector kinetic term, and therefore cannot be positive definite. In [12] the existence of fivedimensional Euclidean vector multiplet actions with a relative sign between scalar and vector term was deduced using dimensional reduction of the bosonic Lagrangians of higherdimensional supergravity theories, and of their Killing spinor equations. We have now derived this result by imposing supersymmetry directly in five dimensions. Our derivation shows in particular that there is no option, because this relative sign is required by

\footnotetext{
${ }^{15}$ In the following it is always understood that we chosen the convention which makes $\theta_{1}$ positive, as explained above.
} 
supersymmetry. We remark that this is a non-trivial insight, since, for instance, it was argued in [12] that an analogous relative sign for four-dimensional Euclidean vector multiplets can be changed by a field redefinition. We will present a full off-shell analysis of the four-dimensional $\mathcal{N}=2$ vector multiplet theories in [22].

Since the supersymmetric Euclidean action is not definite, there is no preferred choice of a sign. We make the conventional choice $s_{\lambda}=-1$ which results in

$$
\begin{aligned}
L_{(0,5)}= & \left(-\frac{1}{4} F_{\mu \nu}^{I} F^{J \mu \nu}+\frac{1}{2} \partial_{\mu} \sigma^{I} \partial^{\mu} \sigma^{J}-\frac{1}{2} \bar{\lambda}^{I} \not \partial \lambda^{J}+Y_{i j}^{I} Y^{i j J}\right) \mathcal{F}_{I J} \\
& +\left(\frac{1}{24} \varepsilon^{\mu \nu \rho \sigma \tau} A_{\mu}^{I} F_{\nu \rho}^{J} F_{\sigma \tau}^{K}-\frac{1}{8} \bar{\lambda}^{I} \gamma^{\mu \nu} F_{\mu \nu}^{J} \lambda^{K}-\frac{1}{2} \bar{\lambda}^{I i} \lambda^{J j} Y_{i j}^{K}\right) \mathcal{F}_{I J K} . \\
\delta A_{\mu}^{I}= & \frac{1}{2} \bar{\epsilon} \gamma_{\mu} \lambda^{I}, \quad \delta \sigma^{I}=\frac{1}{2} \bar{\epsilon} \lambda^{I}, \quad, \delta Y^{i j I}=-\frac{1}{2} \bar{\epsilon}^{\left(i \not \partial \lambda^{j) I}\right.}, \\
\delta \lambda^{i I}= & -\frac{1}{4} \gamma^{\mu \nu} F_{\mu \nu}^{I} \epsilon^{i}+\frac{1}{2} \not \partial \sigma^{I} \epsilon^{i}-Y^{i j I} \epsilon_{j} .
\end{aligned}
$$

\subsubsection{Signature $(5,0)$}

For signature $(5,0)$ we find instead

$$
s_{\sigma}=s_{\lambda}=s_{F}=-s_{Y}= \pm 1 .
$$

Taking into account that the space-time metric is negative definite, the Euclidean action is again indefinite. As for signature $(0,5)$ we make a conventional choice of the overall sign:

$$
\begin{aligned}
L= & \left(-\frac{1}{4} F_{\mu \nu}^{I} F^{J \mu \nu}-\frac{1}{2} \partial_{\mu} \sigma^{I} \partial^{\mu} \sigma^{J}-\frac{1}{2} \bar{\lambda}^{I} \not \partial \lambda^{J}+Y_{i j}^{I} Y^{i j J}\right) \mathcal{F}_{I J} \\
& +\left(\frac{1}{24} \varepsilon^{\mu \nu \rho \sigma \tau} A_{\mu}^{I} F_{\nu \rho}^{J} F_{\sigma \tau}^{K}-\frac{i}{8} \bar{\lambda}^{I} \gamma^{\mu \nu} F_{\mu \nu}^{J} \lambda^{K}-\frac{i}{2} \bar{\lambda}^{I i} \lambda^{J j} Y_{i j}^{K}\right) \mathcal{F}_{I J K} . \\
\delta A_{\mu}^{I}= & \frac{1}{2} \bar{\epsilon} \gamma_{\mu} \lambda^{I}, \quad \delta \sigma^{I}=\frac{i}{2} \bar{\epsilon} \lambda^{I}, \quad \delta Y^{i j I}=-\frac{1}{2} \bar{\epsilon}^{\left(i \not \partial \lambda^{j}\right) I}, \\
\delta \lambda^{i I}= & -\frac{1}{4} \gamma^{\mu \nu} F_{\mu \nu}^{I} \epsilon^{i}-\frac{i}{2} \not \partial \sigma^{I} \epsilon^{i}-Y^{i j I} \epsilon_{j} .
\end{aligned}
$$

\subsubsection{Signature $(2,3)$}

We now turn to the exotic signatures with two time-like directions. In signature $(2,3)$, (4.8) implies

$$
-s_{\sigma}=s_{\lambda}=s_{F}=s_{Y}= \pm 1 .
$$

With a conventional choice of overall sign, we obtain:

$$
\begin{aligned}
L_{(2,3)}= & \left(-\frac{1}{4} F_{\mu \nu}^{I} F^{J \mu \nu}+\frac{1}{2} \partial_{\mu} \sigma^{I} \partial^{\mu} \sigma^{J}-\frac{1}{2} \bar{\lambda}^{I} \not \partial \lambda^{J}-Y_{i j}^{I} Y^{i j J}\right) \mathcal{F}_{I J} \\
& +\left(\frac{1}{24} \varepsilon^{\mu \nu \rho \sigma \tau} A_{\mu}^{I} F_{\nu \rho}^{J} F_{\sigma \tau}^{K}-\frac{1}{8} \bar{\lambda}^{I} \gamma^{\mu \nu} F_{\mu \nu}^{J} \lambda^{K}+\frac{i}{2} \bar{\lambda}^{I i} \lambda^{J j} Y_{i j}^{K}\right) \mathcal{F}_{I J K}, \\
\delta A_{\mu}^{I}= & \frac{1}{2} \bar{\epsilon} \gamma_{\mu} \lambda^{I}, \quad \delta \sigma^{I}=\frac{1}{2} \bar{\epsilon} \lambda^{I}, \quad \delta Y^{i j I}=-\frac{i}{2} \bar{\epsilon}^{\left(i \not \partial \lambda^{j) I}\right.}, \\
\delta \lambda^{i I}= & -\frac{1}{4} \gamma^{\mu \nu} F_{\mu \nu}^{I} \epsilon^{i}+\frac{1}{2} \not \partial \sigma^{I} \epsilon^{i}+i Y^{i j I} \epsilon_{j} .
\end{aligned}
$$




\subsubsection{Signature $(3,2)$}

Finally, in signature $(3,2)$ we find

$$
s_{\sigma}=s_{\lambda}=s_{F}=s_{Y}= \pm 1
$$

and with a conventional choice of overall sign

$$
\begin{aligned}
L_{(3,2)}= & \left(-\frac{1}{4} F_{\mu \nu}^{I} F^{J \mu \nu}-\frac{1}{2} \partial_{\mu} \sigma^{I} \partial^{\mu} \sigma^{J}-\frac{1}{2} \bar{\lambda}^{I} \not \partial \lambda^{J}-Y_{i j}^{I} Y^{i j J}\right) \mathcal{F}_{I J} \\
& +\left(\frac{1}{24} \varepsilon^{\mu \nu \rho \sigma \tau} A_{\mu}^{I} F_{\nu \rho}^{J} F_{\sigma \tau}^{K}-\frac{i}{8} \bar{\lambda}^{I} \gamma^{\mu \nu} F_{\mu \nu}^{J} \lambda^{K}-\frac{1}{2} \bar{\lambda}^{I i} \lambda^{J j} Y_{i j}^{K}\right) \mathcal{F}_{I J K}, \\
\delta A_{\mu}^{I}= & \frac{1}{2} \bar{\epsilon} \gamma_{\mu} \lambda^{I}, \quad \delta \sigma^{I}=\frac{i}{2} \bar{\epsilon} \lambda^{I}, \quad \delta Y^{i j I}=-\frac{i}{2} \bar{\epsilon}^{(i} \not \partial \lambda^{j) I}, \\
\delta \lambda^{i I}= & -\frac{1}{4} \gamma^{\mu \nu} F_{\mu \nu}^{I} \epsilon^{i}-\frac{i}{2} \not \partial \sigma^{I} \epsilon^{i}+i Y^{i j I} \epsilon_{j} .
\end{aligned}
$$

While the interpretation of relative and overall signs is not obvious for a theory with multiple time-like directions, we observe that all relative sign flips between signatures $(2,3)$ and $(3,2)$ can be interpreted as going from a mostly plus to a mostly minus convention for the space-time metric, together with factors $i$ to account for the reality properties of spinor bilinears.

We finally note that by comparing our results signature by signature to [13] it is straightforward to check that they agree where comparable, that is for the bosonic terms of the Lagrangians and the supersymmetry variations of the fermions.

\section{Open problems and outlook}

In this paper we have taken the first step to developing a formalism which allows us to construct supersymmetric theories simultanously for all space-time signatures. We have restricted ourselves to supersymmetry algebras based on complex irreducible spinor representations, where we determined the possible R-symmetry groups. In a companion paper we will extend this analysis to the general case [19]. As an application we constructed off-shell vector multiplets and the associated supersymmetric Lagrangians for the unique minimal five-dimensional supersymmetry algebra in arbitrary signature. The five dimensional case is particularly straightforward to fully analyze, because (i) Dirac spinors are complex irreducible, (ii) Majorana conditions do not lead to a non-trivial smaller supersymmetry algebra, and (iii) the space of superbrackets is one-dimensional. The natural next application, rigid four-dimensional $\mathcal{N}=2$ vector multiplets, to be presented in [22], is much richer. Four-dimensional Dirac spinors are complex reducible, and in some signatures a Majorana condition defines a smaller supersymmetry algebra. Moreover, from [15] one can read off that the space of $\mathcal{N}=2$ superbrackets is four-dimensional, and it is not clear a priori whether all these superbrackets define isomorphic Lie superalgebras. The scalar geometry of four-dimensional vector multiplets depends on the signature: it is special Kähler in Lorentz signature, but special para-Kähler in Euclidean signature [6]. This makes the 
issues concerning relative signs between kinetic terms in the Lagrangian much more interesting. In [12] it was observed that by dimensional reduction one can arrive at two different formulations of the bosonic sector of four-dimensional Euclidean vector multiplets, which differ by a relative sign flip between the scalar and the vector term. While it was shown in [12] that both formulations of the bosonic sector are related by a field redefinition, it remains to be seen whether and how this extends to the fermionic terms and supersymmetry transformations. Also according to [13] the reduction of exotic five-dimensional theories to Lorentzian signature gives rise to non-standard signs for some kinetic terms, similar to type II* theories in ten dimensions. In [22] we will provide a complete analysis, based on a further development of the formalism presented in this paper. Other directions which we will explore is the application of our formalism to other multiplets, notably hypermultiplets and the Weyl multiplet. The later will allow us to construct off-shell realizations of $\mathcal{N}=2$ supergravity in arbitrary signature.

\section{Acknowledgments}

T.M. thanks Vicente Cortés for many useful discussions and the Department of Mathematics and Centre for Mathematical Physics of the University of Hamburg for support and hospitality during various stages of this work. The work of T.M. was partly supported by the STFC consolidated grant ST/G00062X/1. The work of L.G. was supported by STFC studentship ST/1643452.

\section{A Spinor conventions}

We use the same conventions for spinor indices as in [6]. Dirac spinors $\psi \in \mathbb{S}$ have lower indices, $\psi=\left(\psi_{\alpha}\right)$. $\gamma$ matrices are endomorphisms on the spinor module, $\gamma^{\mu}=\left(\gamma_{\alpha}^{\mu}{ }^{\beta}\right)$. The matrices $A$ and $C$ represent a sesquilinear form and a bilinear form on $\mathbb{S}: A=\left(A^{\alpha \beta}\right)$, and $C=\left(C^{\alpha \beta}\right)$. The inverse matrices are denoted $A^{-1}=\left(A_{\alpha \beta}\right)$, and $C^{-1}=\left(C_{\alpha \beta}\right)$. Note that the definition $A=\gamma_{1} \cdots \gamma_{t}$ is an equation between matrices, not between maps. Therefore this identification holds with respect to a fixed basis for the spinor module. However, all expressions appearing in the Lagrangian and in the supersymmetry variations are covariant with respect to Lorentz transformations, since all spinor and other indices are properly contracted. Therefore any result obtained in our distinguished coordinate system holds in all coordinate systems.

Indices on Dirac spinors are raised and lowered using $A$ and $A^{-1}$ :

$$
\lambda^{\alpha}=A^{\alpha \beta} \lambda_{\beta} \Leftrightarrow \lambda_{\alpha}=A_{\alpha \beta} \lambda^{\beta} .
$$

Spinor indices on doubled spinors $\left(\lambda_{\alpha}^{i}\right) \in \mathbb{S} \otimes \mathbb{C}^{2}$ are raised and lowered using $C$ and $C^{-1}$ :

$$
\lambda^{i \alpha}=C^{\alpha \beta} \lambda_{\beta}^{i} \Leftrightarrow \lambda_{\alpha}^{i}=C_{\alpha \beta} \lambda^{i \beta} .
$$

Internal indices $i, j=1,2$ on doubled spinors are raised and lowered according to

$$
\lambda^{i}=\varepsilon^{i j} \lambda_{j} \Leftrightarrow \lambda_{i}=\lambda^{j} \varepsilon_{j i},
$$


where $\varepsilon_{i j}=-\varepsilon_{j i}$ and $\varepsilon^{i j} \varepsilon_{k j}=\delta_{k}^{i}=-\varepsilon^{i j} \varepsilon_{j k}$. Note that $\left(\varepsilon^{i j}\right)=-\left(\varepsilon_{i j}\right)^{-1}$, which makes raising and lowering $\mathrm{SU}(2)$ indices consistent with the NW-SE convention. In contrast, our convention for raising and lowering spinor indices $\alpha, \beta, \ldots$ does not comply with the NW-SE convention.

As an application we extract the structure constants of the supersymmetry algebra from the associated vector-valued sesquilinear form. The natural vector-valued sesquilinear form on the spinor module is

$$
A\left(\gamma^{\mu} \lambda, \chi\right)=\lambda_{\alpha}^{*}\left(\gamma^{\dagger}\right)_{\beta}^{\mu \alpha} A^{\beta \gamma} \chi_{\gamma}=\lambda^{* \alpha} \gamma_{\alpha}^{\mu}{ }^{\beta} A_{\beta \gamma} \chi^{\beta} .
$$

Therefore the matrix representing $A\left(\gamma^{\mu} \cdot, \cdot\right)$ with respect to the dual ('upper index') coordinates $\lambda^{\alpha}, \chi^{\beta}$ is

$$
K[A]_{\alpha \beta}^{\mu}=\left(\gamma^{\mu} A^{-1}\right)_{\alpha \beta} .
$$

The vector-valued real bilinear form defining the supersymmetry algebra in terms of Dirac spinors is the real or imaginary part of $A\left(\gamma^{\mu} \cdot, \cdot\right)$, depending on signature. Let us take the case where it is the real part, for definiteness. The anticommutator of a general linear combination of supercharges is defined using the vector-valued bilinear form by

$$
\left\{\lambda^{\alpha} Q_{\alpha}, \chi^{\beta} Q_{\beta}\right\}=A\left(\gamma^{\mu} \lambda, \chi\right) P_{\mu}=\lambda^{\alpha} \chi^{\beta} \operatorname{Re}\left(\gamma^{\mu} A^{-1}\right)_{\alpha \beta} P_{\mu},
$$

from which we obtain

$$
\left\{Q_{\alpha}, Q_{\beta}\right\}=\operatorname{Re}\left(\gamma^{\mu} A^{-1}\right)_{\alpha \beta} P_{\mu} \varepsilon_{i j} .
$$

Similarly, the bilinear form on the doubled spinor module is

$$
C\left(\gamma^{\mu} \lambda, \chi\right)=-\frac{1}{2} \lambda_{\alpha}^{i}\left(\gamma^{\mu T}\right)_{\beta}^{\alpha} C^{\beta \gamma} \chi_{\gamma}^{j} \varepsilon_{j i}=-\frac{1}{2} \lambda^{i \alpha} \chi^{j \beta}\left(\gamma^{\mu} C^{-1}\right)_{\alpha \beta} \varepsilon_{i j} .
$$

Substituting this into

$$
\left\{\lambda^{i \alpha} Q_{i \alpha}, \chi^{j \beta} Q_{j \beta}\right\}=C\left(\gamma^{\mu} \lambda, \chi\right) P_{\mu}
$$

we obtain

$$
\left\{Q_{i \alpha}, Q_{j \beta}\right\}=-\frac{1}{2}\left(\gamma^{\mu} C^{-1}\right)_{\alpha \beta} P_{\mu} \varepsilon_{i j}
$$

\section{B Para-quaternions}

The para-quaternions $q \in \mathbb{H}^{\prime}$ are defined by

$$
\mathbb{H}^{\prime}=\left\{q=q_{0}+q_{1} e_{1}+q_{2} e_{2}+q_{3} e_{12} \mid q_{0}, q_{1}, q_{2}, q_{3} \in \mathbb{R}\right\},
$$

where

$$
e_{1}^{2}=e_{2}^{2}=1, \quad e_{12}:=e_{1} e_{2}=-e_{2} e_{1} \Rightarrow e_{12}^{2}=-1 .
$$

They form a four-dimensional real associative algebra. One defines the conjugate

$$
q^{*}=q_{0}-q_{1} e_{1}-q_{2} e_{2}-q_{3} e_{12}
$$

and the norm

$$
N(q)=q q^{*}=q_{0}^{2}-q_{1}^{2}-q_{2}^{2}+q_{3}^{2}
$$


A para-quaternion is invertible iff its norm is non-zero, with inverse

$$
q^{-1}=\frac{q^{*}}{N(q)}
$$

Note that in contrast to the quaternions, the para-quaternions do not form a skew-field. As a normed algebra, $\mathbb{H}^{\prime}$ is isomorphic to algebra $\mathbb{R}(2)$ of real $2 \times 2$ matrices, with the norm provided by the determinant. An isomorphism is given by

$$
1 \mapsto\left(\begin{array}{ll}
1 & 0 \\
0 & 1
\end{array}\right), \quad e_{1} \mapsto\left(\begin{array}{ll}
0 & 1 \\
1 & 0
\end{array}\right), \quad e_{2} \mapsto\left(\begin{array}{cc}
1 & 0 \\
0 & -1
\end{array}\right), \quad e_{12} \mapsto\left(\begin{array}{cc}
0 & -1 \\
1 & 0
\end{array}\right),
$$

so that

$$
q \mapsto M(q)=\left(\begin{array}{ll}
q_{0}+q_{2} & q_{1}-q_{3} \\
q_{1}+q_{3} & q_{0}-q_{2}
\end{array}\right) .
$$

Note that $\operatorname{det} M(q)=q q^{*}=N(q)$.

Therefore the group $\left(\mathbb{H}^{\prime}\right)^{*}$ of invertible para-quaternions is

$$
\left(\mathbb{H}^{\prime}\right)^{*}:=\mathrm{GL}\left(1, \mathbb{H}^{\prime}\right):=\{q \in \mathbb{H} \mid N(q) \neq 0\} \simeq \mathrm{GL}(2, \mathbb{R})
$$

and the subgroup of unit norm para-quaternions is

$$
\mathrm{U}\left(1, \mathbb{H}^{\prime}\right):=\left\{q \in \mathbb{H}^{*} \mid N(q)=1\right\} \simeq \mathrm{SL}(2, \mathbb{R})
$$

Para-quaternions can be viewed as pairs of complex numbers,

$$
q=\left(q_{0}+e_{12} q_{3}\right)+e_{1}\left(q_{1}+e_{12} q_{2}\right)=: u+e_{1} v,
$$

where $u, v$ are interpreted as complex numbers by identifying $e_{12} \simeq i$.

The normed algebra $\mathbb{H}^{\prime}$ can be represented by complex $2 \times 2$ matrices:

$$
q \mapsto \tilde{M}(q)=\left(\begin{array}{cc}
u & v \\
v^{*} & u^{*}
\end{array}\right) \in \mathbb{C}(2) .
$$

Note that $N(q)=u u^{*}-v v^{*}=\operatorname{det} \tilde{M}$. The subgroup of unit norm matrices is $\operatorname{SU}(1,1) \simeq$ $\mathrm{SL}(2, \mathbb{R}) \simeq \mathrm{U}\left(1, \mathbb{H}^{\prime}\right)$.

Matrices of the form $\tilde{M}(q)$ are invariant under the real structure on $\mathbb{C}(2)$ defined by

$$
\rho: \tilde{M} \mapsto \eta \tilde{M}^{*} \eta, \quad \eta=\left(\begin{array}{ll}
0 & 1 \\
1 & 0
\end{array}\right) .
$$

Thus matrices of the form $\tilde{M}(q)$ form a subalgebra isomorphic to $\mathbb{R}(2)$, as they must:

$$
\mathbb{H}^{\prime} \simeq\{\tilde{M} \in \mathbb{C}(2) \mid \rho(\tilde{M})=\tilde{M}\} \simeq \mathbb{R}(2) .
$$

Further note that $\mathbb{H}^{\prime} \simeq C l_{1,1} \simeq C l_{0,2}$ as real associative algebras. 


\section{Quaternions}

For comparison let us also review standard results about the quaternions $\mathbb{H}$. The quaternions $q \in \mathbb{H}$ are defined by

$$
\mathbb{H}=\left\{q=q_{0}+q_{1} i+q_{2} j+q_{3} k \mid q_{0}, q_{1}, q_{2}, q_{3} \in \mathbb{R}\right\},
$$

where

$$
i^{2}=j^{2}=-1, \quad k=i j=-j i \Rightarrow k^{2}=-1 .
$$

They form a four-dimensional real associative algebra, One defines the conjugate

$$
q^{*}=q_{0}-q_{1} i-q_{2} j-q_{3} k
$$

and the $\left(\right.$ reduced $\left.^{16}\right)$ norm

$$
N(q)=q q^{*}=q_{0}^{2}+q_{1}^{2}+q_{2}^{2}+q_{3}^{2} .
$$

A quaternion is invertible iff

$$
N(q) \neq 0 \Leftrightarrow q \neq 0,
$$

with inverse

$$
q^{-1}=\frac{q^{*}}{N(q)}
$$

Since only $q=0$ does not have an inverse, the quaternions do not only form an associative algebra, but a skew field. As a normed algebra, $\mathbb{H}$ is isomorphic to the matrix algebra

$$
\left\{\left(\begin{array}{cc}
u & v \\
-v^{*} & u^{*}
\end{array}\right) \mid u, v \in \mathbb{C}\right\} \subset \mathbb{C}(2) .
$$

An isomorphism is provided by

$$
\begin{aligned}
q & \mapsto M(q)=q_{0} \mathbb{1}+q_{1} i \sigma^{1}+q_{2} i \sigma^{2}+q_{3} i \sigma^{3}=\left(\begin{array}{cc}
q_{0}+i q_{3} & q_{2}+i q_{1} \\
-q_{2}+i q_{1} & q_{0}-i q_{3}
\end{array}\right) \\
& =\left(\begin{array}{cc}
u & v \\
-u^{*} & v^{*}
\end{array}\right),
\end{aligned}
$$

where $\sigma^{i}, i=1,2,3$ are the Pauli matrices and where $u=q_{0}+i q_{3}$ and $v=q_{2}+i q_{1}$. The norm of $q$ is equal to the determinant of the corresponding complex $2 \times 2$ matrix:

$$
N(q)=a^{2}+b^{2}+c^{2}+d^{2}=u u^{*}+v v^{*}=\operatorname{det} M(q) .
$$

If we impose unit norm, then

$$
N(q)=u u^{*}+v v^{*}=1,
$$

\footnotetext{
${ }^{16}$ For the quaternion one often refers to $\|q\|:=N(q)^{1 / 2}$ as the norm.
} 
and $M(q) \in \mathrm{SU}(2)$. Therefore the group of unit norm quaternions is

$$
\mathrm{U}(1, \mathbb{H})=\{q \mid N(q)=1\} \simeq \mathrm{SU}(2) .
$$

Any matrix $M(q)$ with $N(q) \neq 0$ can be written as a positive scalar multiple of an $\mathrm{SU}(2)$ matrix, and therefore the group of invertible quaternions is

$$
\mathbb{H}^{*}=\mathrm{GL}(1, \mathbb{H})=\{q \in \mathbb{H} \mid N(q) \neq 0\}=\mathbb{R}^{>0} \times \mathrm{SU}(2)
$$

Further note that $\mathbb{H} \simeq C_{2,0}$ as real associative algebras.

Open Access. This article is distributed under the terms of the Creative Commons Attribution License (CC-BY 4.0), which permits any use, distribution and reproduction in any medium, provided the original author(s) and source are credited.

\section{References}

[1] C.M. Hull, Timelike $T$ duality, de Sitter space, large $N$ gauge theories and topological field theory, JHEP 07 (1998) 021 [hep-th/9806146] [INSPIRE].

[2] C.M. Hull, Duality and the signature of space-time, JHEP 11 (1998) 017 [hep-th/9807127] [INSPIRE].

[3] R. Dijkgraaf, B. Heidenreich, P. Jefferson and C. Vafa, Negative Branes, Supergroups and the Signature of Spacetime, JHEP 02 (2018) 050 [arXiv: 1603.05665] [INSPIRE].

[4] C.M. Hull and B. Julia, Duality and moduli spaces for timelike reductions, Nucl. Phys. B 534 (1998) 250 [hep-th/9803239] [INSPIRE].

[5] E. Cremmer, I.V. Lavrinenko, H. Lü, C.N. Pope, K.S. Stelle and T.A. Tran, Euclidean signature supergravities, dualities and instantons, Nucl. Phys. B 534 (1998) 40 [hep-th/9803259] [INSPIRE].

[6] V. Cortes, C. Mayer, T. Mohaupt and F. Saueressig, Special geometry of Euclidean supersymmetry. I. Vector multiplets, JHEP 03 (2004) 028 [hep-th/0312001] [INSPIRE].

[7] V. Cortes, C. Mayer, T. Mohaupt and F. Saueressig, Special geometry of euclidean supersymmetry. II. Hypermultiplets and the c-map, JHEP 06 (2005) 025 [hep-th/0503094] [INSPIRE].

[8] V. Cortes and T. Mohaupt, Special Geometry of Euclidean Supersymmetry III: The local r-map, instantons and black holes, JHEP 07 (2009) 066 [arXiv: 0905.2844] [INSPIRE].

[9] V. Cortés, P. Dempster, T. Mohaupt and O. Vaughan, Special Geometry of Euclidean Supersymmetry IV: the local c-map, JHEP 10 (2015) 066 [arXiv: 1507.04620] [INSPIRE].

[10] J.B. Gutowski and W.A. Sabra, Euclidean $N=2$ Supergravity, Phys. Lett. B 718 (2012) 610 [arXiv: 1209.2029] [INSPIRE].

[11] W. Sabra and O. Vaughan, $10 D$ to $4 D$ Euclidean Supergravity over a Calabi-Yau three-fold, Class. Quant. Grav. 33 (2016) 015010 [arXiv:1503.05095] [INSPIRE].

[12] W.A. Sabra and O. Vaughan, Euclidean Supergravity in Five Dimensions, Phys. Lett. B 760 (2016) 14 [arXiv: 1603.09244] [INSPIRE]. 
[13] W.A. Sabra, Special geometry and space-time signature, Phys. Lett. B 773 (2017) 191 [arXiv: 1706.05162] [INSPIRE].

[14] B. de Wit and V. Reys, Euclidean supergravity, JHEP 12 (2017) 011 [arXiv:1706.04973] [INSPIRE].

[15] D.V. Alekseevsky and V. Cortés, Classification of n-(super)-extended poincare algebras and bilinear invariants of spinor representations of spin $(p, q)$, Commun. Math. Phys. 183 (1997) 477.

[16] E. Bergshoeff and A. Van Proeyen, The many faces of OSp(1-32), Class. Quant. Grav. 17 (2000) 3277 [hep-th/0003261] [INSPIRE].

[17] E.A. Bergshoeff, J. Hartong, A. Ploegh, J. Rosseel and D. Van den Bleeken, Pseudo-supersymmetry and a tale of alternate realities, JHEP 07 (2007) 067 [arXiv: 0704.3559] [INSPIRE].

[18] D.V. Alekseevsky, V. Cortes, C. Devchand and A. Van Proeyen, Polyvector superPoincaré algebras, Commun. Math. Phys. 253 (2004) 385 [hep-th/0311107] [INSPIRE].

[19] L. Gall and T. Mohaupt, R-symmetry groups for any signature, in preparation.

[20] H.B. Lawson and M.L. Michelsohn, Spin geometry, Princeton University Press, (1989).

[21] A. Van Proeyen, Tools for supersymmetry, Ann. U. Craiova Phys. 9 (1999) 1 [hep-th/9910030] [INSPIRE].

[22] V. Cortés, L. Gall and T. Mohaupt, Four-dimensional vector multiplets in any signature, in preparation.

[23] R. Gilmore, Lie groups, lie algebras and some of their applications, A. Wiley Interscience Publication, (1974).

[24] D.Z. Freedman and A. Van Proeyen, Supergravity, Cambridge University Press, (2012).

[25] E. Bergshoeff, T. de Wit, R. Halbersma, S. Cucu, M. Derix and A. Van Proeyen, Weyl multiplets of $N=2$ conformal supergravity in five-dimensions, JHEP 06 (2001) 051 [hep-th/0104113] [INSPIRE].

[26] T. Mohaupt and K. Waite, Instantons, black holes and harmonic functions, JHEP 10 (2009) 058 [arXiv: 0906.3451] [INSPIRE].

[27] T. Mohaupt and K. Waite, Euclidean Actions, Instantons, Solitons and Supersymmetry, J. Phys. A 44 (2011) 175403 [arXiv: 1011.6301] [INSPIRE]. 